\title{
The influence of variability and defects on the mechanical performance of tailorable composites
}

\author{
James M. Finley ${ }^{\mathrm{a}}$, Joël Henry ${ }^{\mathrm{a}}$, Milo S.P. Shaffer ${ }^{\mathrm{b}}$, Soraia Pimenta ${ }^{\mathrm{a}, *}$ \\ ${ }^{a}$ Department of Mechanical Engineering, South Kensington Campus, Imperial College London, SW $2 A Z$, \\ United Kingdom \\ ${ }^{b}$ Department of Chemistry, South Kensington Campus, Imperial College London, SWr 2AZ, United \\ Kingdom
}

\begin{abstract}
Aligned hybrid-fibre discontinuous composites offer the ability to tailor their mechanical response through careful microstructural design. However, with tailorability comes microstructural complexity, which in turn leads to many sources of variability and defects. A virtual testing framework was further extended to investigate the influence of variability and defects on the mechanical performance of various aligned discontinuous composite material systems. This approach identified the most critical sources of variability as (i) fibre strength, (ii) the distance between fibre ends, or (iii) the level of fibre-type intermingling, depending on the material system. Fibre vacancy defects were shown to have the most significant influence on the strength and ductility of aligned discontinuous composites, although this sensitivity can be reduced through hybridisation of the fibre types.
\end{abstract}

Keywords: aligned discontinuous composites, variability, defects, fracture

\section{Introduction}

Composite materials are widely used in the aerospace industry due to their high specific strength and specific stiffness [1]. However, like most materials, composites are sensitive to defects and variability. Various authors have shown that the performance of continuousfibre composite materials is negatively influenced by variability in inter-fibre distance (or local fibre volume fraction) [2, 3, fibre strength and Young's modulus [4, 5], and matrix interfacial shear strength [6, 7]. Defects such as fibre misalignment [8] and voidage [9, 10] have also been shown to significantly influence the mechanical performance of composites.

The effects of variability and defects in continuous fibre composites have been modelled

\footnotetext{
${ }^{*}$ Corresponding author.

Email address: soraia.pimenta@imperial.ac.uk (Soraia Pimenta)
} 


\section{Nomenclature}

\begin{tabular}{ll}
\multicolumn{2}{l}{ General properties } \\
$n_{*}$ & number of $(*)$ \\
$v_{\mathrm{f}}$ & fibre volume fraction \\
$v_{\mathrm{c}}$ & carbon ratio \\
$\phi$ & fibre diameter \\
$t_{*}$ & thickness of $(*)$ \\
$l_{*}$ & length of $(*)$ \\
$\bar{*}$ & mean of $(*)$ \\
$C_{o} V_{*}$ & coefficient of variation of $(*)$ \\
$\zeta_{\mathrm{rf}}$ & fraction of fragmentation defects \\
$\zeta_{\mathrm{ri}}$ & fraction of interface defects \\
$\zeta_{\mathrm{fv}}$ & fraction of fibre vacancy defects \\
$m$ & Weibull modulus \\
$l_{\mathrm{w}}$ & Weibull gauge length \\
$E_{\mathrm{f}}$ & fibre Young's modulus \\
$X_{\mathrm{f}}$ & fibre reference strength \\
$S_{\mathrm{m}}$ & average matrix shear strength \\
$G_{\mathrm{m}}$ & matrix shear modulus \\
$\mathcal{G}_{\mathrm{I}}$ & mode-I fracture toughness \\
$\mathcal{G}_{\mathrm{II}}$ & mode-II fracture toughness \\
$\tau_{\mu}^{0}$ & pull-out frictional stress \\
$\delta l$ & fibre-fibre overlap length \\
$\boldsymbol{T}$ & matrix of fibre types \\
$\mathcal{U}$ & random distribution sample \\
$\mathcal{N}$ & normal distribution sample \\
$\mathbb{E}$ & expected value
\end{tabular}

\begin{tabular}{ll}
\multicolumn{2}{l}{ Subscripts / superscripts } \\
$\mathrm{f}$ & fibre \\
$\mathrm{c}$ & carbon fibre \\
$\mathrm{g}$ & glass fibre \\
$\mathrm{i}$ & fibre row within specimen \\
$\mathrm{j}$ & fibre column within specimen \\
$\mathrm{RVE}$ & representative volume element \\
$\mathrm{r}$ & RVE index \\
$\mathrm{s}$ & specimen \\
$\mathrm{k}$ & cluster index \\
$\varepsilon$ & strain increment \\
$(*)^{\prime}$ & critical value of $(*)$
\end{tabular}

\author{
Inter-fibre distance variables \\ $t_{\mathrm{i}} \quad$ inter-fibre distance \\ $t_{\mathrm{a}} \quad$ fibre centre distance \\ $p \quad$ areal density \\ erfc residual error function \\ $N \quad$ neighbouring fibre index \\ $N * \quad$ no. of neighbouring fibres \\ $\alpha \quad$ load sharing coefficient
}

\section{Damage event analysis}

$\begin{array}{ll}n_{\mathrm{e}_{\mathrm{s}}} & \text { no. softening events } \\ n_{\mathrm{e}_{\mathrm{d}}} & \text { no. debonding events } \\ n_{\mathrm{e}_{\mathrm{f}}} & \text { no. fibre fragmentation events } \\ n_{\mathrm{e}_{\mathrm{ff}}} & \text { no. fibre failure events }\end{array}$

Stress, strain, and fracture variables

$\sigma \quad$ matrix of stresses

$\sigma_{\mathrm{o}} \quad$ cluster opening stress

$\epsilon \quad$ matrix of strains

$X_{\text {RVE }} \quad$ RVE ultimate strength

$\boldsymbol{A}_{\mathrm{f}} \quad$ matrix of fibre areas

$\boldsymbol{A}_{\mathrm{f}_{\mathrm{b}}} \quad$ matrix of damaged fibre areas

$\boldsymbol{A}_{\mathrm{f}_{\mathrm{p}}} \quad$ matrix of undamaged fibre areas

$\boldsymbol{E}$ initial fibre stiffness matrix (no defects)

$E_{\mathrm{RVE}} \quad$ RVE initial stiffness

$D$ matrix fo fibre damage states

d damage threshold

$\boldsymbol{B}$ matrix of damaged fibres

C matrix of clusters

$\boldsymbol{n}_{\mathrm{k}} \quad$ no. clusters of damaged fibres

$\boldsymbol{a}_{\mathrm{e}} \quad$ cluster equivalent crack sizes

$\boldsymbol{J} \quad$ cluster strain energy release rates

$\boldsymbol{R}_{\text {min }}$ matrix of min. reserve factors

$\boldsymbol{K}$ critical cluster within the cross-section

at the micro-mechanical [2, 11, 12, 13, macro-mechanical [5, 14], and structural levels [15]. 
approach is required if local damage and fracture [16] or microscopic defects [17] are to be simulated. However, the micro-mechanical approach is often prohibitively computationally intensive, especially if stochastic features such as random spatial distributions of fibres [2, 12, 13 and stochastic material properties are included; therefore, in most cases the micromechanical approach has only been used to capture the behaviour of small regions of the composite. Moreover, when using micro-mechanical models over a small region of a composite, the properties must then be homogenised across different length scales, which often leads to errors [11.

Aligned discontinuous composites (ADCs) [18] are a sub-class of fibre-reinforced composites that feature tailorable mechanical properties, which can be controlled by manipulation of the microstructure. For example, short-fibre ADCs (with fibre lengths of approximately $0.5 \mathrm{~mm}$ and an epoxy matrix) exhibit a pseudo-ductile response due to non-linear matrix behaviour or interfacial damage initiated at the fibre-ends, while longfibre ADCs (with fibre lengths of approximately $6.0 \mathrm{~mm}$ and an epoxy matrix) are stronger and stiffer because the longer fibres are able to recover more load through shear of the matrix [19, 20, 21]. The transition between a pseudo-ductile response and stronger, more brittle response has been determined to be at a fibre length of around $1.1 \mathrm{~mm}$ [19], although this calculation depends on the combination of constituent properties used and the sources of variability that are considered in the analysis. Hybridisation of different fibretypes offers additional mechanisms for structural tailorability through the combination of fibres with different failure strains $[22,23,24,25,26,27,28]$; the hybridisation technique is particularly successful when applied to ADCs, where the highest levels of fibre-type intermingling can be achieved [25].

While the tailorability of ADCs is very promising for a wide range of applications, the additional complexity in the microstructure leads to more sources of variability and defects than in conventional non-hybrid continuous-fibre composites. Examples of additional sources of variability may include differences in the longitudinal distance between neighbouring fibre-ends, matrix strength variability, and a random hybrid fibre-type arrangement 25]. ADCs may also feature additional sources of defects when compared to continuous-fibre composites, such as poorer fibre alignment, residual fibre fragmentations or interface contamination (due to extra handling), or larger variations in fibre content (or fibre vacancies / voidage).

There has been no accurate yet efficient assessment of how variability and defects may 

ticular. This lack of research is partially down to the complexity of the problem, since predicting final failure of composites requires knowledge of how sub-critical damage at the fibre-level propagates to cause failure of the complete specimen / structure. Moreover, many model runs are required to form a statistically relevant analysis and fully quantify how stochastic effects (such as variability and defects) affect the properties of these materials.

This paper aims to explore how variability and defects affect the strength, stiffness, and ductility of aligned discontinuous composites with non-hybrid or hybrid fibre reinforcement. This aim will be achieved through the use of an accurate yet efficient virtual testing the macroscopic response, without inaccurate homogenisation techniques. Section 2 will describe the virtual testing framework, with the novel developments required to represent all relevant sources of variability and defects detailed in Section 2.2.1 and Section 2.2.2. Section 2.2.3 will describe how the VTF has been developed to handle combinations of of pre-calculated results. Additionally, a new, generalised fracture criterion will be developed in Section 2.2 .5 to accurately capture the influence of defects and variability on the final failure of any ADC microstructure. The results of the study into variability will first be presented and discussed in Section 3 , followed by the results and discussion of the study into defects in Section 4. Finally, conclusions will be drawn in Section 5 .

\section{Model development}

\subsection{Overview of the virtual testing framework}

The virtual testing framework, or VTF [19, 23, 24, 25], is used to simulate the response of aligned discontinuous composite virtual specimens (shown in Figure 1) under tensile loading. The virtual specimen stress-strain response is calculated by bridging the hierarchy of scales within the composite microstructure:

1. The stress-strain profile of each overlap between a pair of neighbouring fibres is calculated using a hybrid non-linear shear-lag model 23, and then combined in series to form the stress-strain response of a single fibre-fibre interaction (Figure 1a).

2. A co-ordination number of $N^{*}=4$ is assumed for the interaction between fibres (with the exception of the virtual specimen edges). Consequently, four neighbouring 
fibre interactions (labelled $N=1,2,3$, and 4 in Figure $1 \mathrm{~b}$ ) are combined in parallel to create the stress-strain response of a single fibre.

3. All fibres within each representative volume element (RVE, which contains the same number of fibres in its cross-section as an equivalently-sized physical specimen) are modelled as per point 2. The RVE splits the specimen into segments with equivalent length to that of the fibres, enabling efficient parallelisation of calculations whilst directly capturing the mechanical response of each fibre-fibre overlap. Their stressstrain responses are then combined in parallel (Figure 1c). Within each RVE, a fibre is identified by its position in the $x$-y plane, using matrix indices $i$ (in the $y$-direction) and $j$ (in the $x$-direction).

4. All RVEs are analysed as per point 3, and their stress-strain responses are combined in series to give the stress-strain curve of the virtual specimen (Figure 1d).

5. Final failure of the virtual specimen is determined by a new non-linear fracture mechanics failure criterion (details of this failure criterion are shown in Section 2.2.5).

The VTF makes no distinction between the matrix and the fibre-matrix interface; instead, the shear-lag strength and toughness for the analysis of each overlap are taken as the weaker of the matrix or the fibre-matrix interface. The term 'matrix' therefore refers to the weaker of the matrix or the fibre-matrix interface for the remainder of this paper.

The fracture behaviour and non-linearity of the matrix are captured using a piecewise linear constitutive law in the shear-lag overlap model (see point 1 above). All fibres in the VTF are linear-elastic, each with a different stochastic strength (defined by a Weibull distribution); fibre fragmentation occurs when the stress in a fibre exceeds its stochastic strength, resulting in a reduction in the number of active neighbouring fibre interactions $\left(N^{*}\right)$, the creation of shorter fibre segments, and the creation of shorter shear-lag overlaps. The VTF can model hybrid [24, 23, 25] (whereby each fibre-type has a different Young's modulus, a different diameter, and a different stochastic strength distribution) or nonhybrid [19] aligned discontinuous fibre reinforcements. For the sake of simplicity, this paper features only high-modulus carbon / E-glass hybrid ADCs; however, any combination of fibre-types can be used, simply by replacing the fibre property inputs to the VTF.

All fibres in each virtual specimen are assumed to have equal length $\left(l_{\mathrm{f}}\right)$ at the start of the simulation, and the random location of the fibre ends is modelled according to a uniform random distribution. The fibre-type arrangement of hybrid ADCs can be modelled by randomly intermingling the fibre-types 24] using a uniform random distribution, or by 
specifying the fibre-type arrangement [25]. The cross-section of the virtual specimen can form a rectangle with any aspect ratio, and the number of fibres in the virtual specimen can be selected to match that of a real tensile specimen.

\subsection{Novel features of the virtual testing framework}

\subsubsection{Modelling novel sources of variability}

The VTF is extended in this work, in order to capture the influence of all relevant sources of variability to be analysed in this paper; these changes are described in detail below:

- Variability of the fibre/matrix interface and local matrix properties have been shown to play a key role in composite strength [6]. Matrix shear strength variability was measured from single fibre pull-out data [29] and fitted to a Weibull distribution (see Table 11); a Weibull distribution was selected as this accurately captures the probability of a single failure event (in this case, the matrix softening / debonding of the matrix) due to weak regions in the matrix. Matrix shear strength variability is added to the VTF by sampling the shear strength of the matrix constitutive law for each interaction from this Weibull distribution.

- Differences in fibre diameter, misalignment of the fibre crystal structure, and fibre composition can all lead to variability in fibre stiffness [30]. Fibre stiffness variability was measured using T-300 single fibre tensile test data [29] and fitted to a Normal distribution; fibre stiffness is the result of the average properties of the whole fibre, meaning that the central limit theorem [31] can be applied, hence justifying the use of the Normal distribution in this case. It is assumed that the coefficient of variation in fibre stiffness remained similar for the T-300 fibres and all fibre-types used in the present study (see Table 1). Fibre stiffness variability is then added to the VTF by sampling the Young's modulus of each fibre in each RVE from a Normal distribution with the determined coefficient of variation, with an expected value scaled to match the corresponding fibre type (see Table 1).

- Variability in inter-fibre distance $\left(t_{\mathrm{i}_{N}}\right.$, as shown in Figure $1 \mathrm{~b}$, where $N \in\{1,2,3,4\}$ denotes the $N^{\text {th }}$ neighbouring fibre surrounding a central fibre) has been shown to locally change the stress concentrations that act on fibres that neighbour another fibre-end or -break [32, 33, 34]. Variability in the inter-fibre distance is captured 


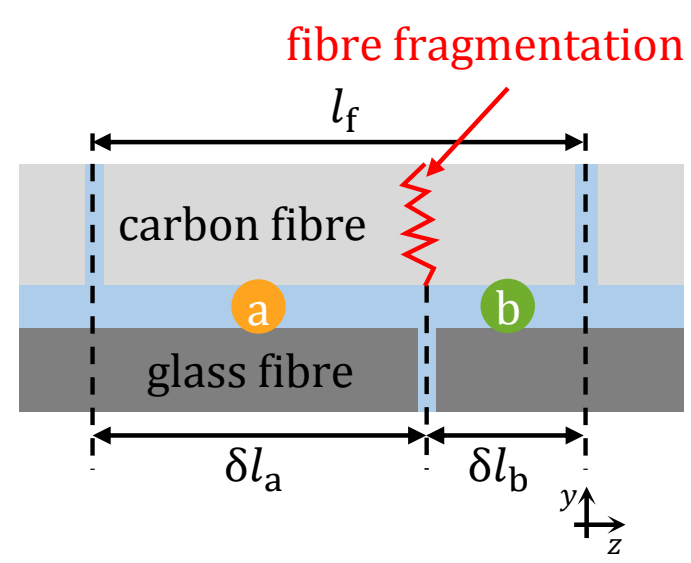

(a) The stress-strain response of overlaps $a$ and $b$ are combined in series to give the stress-strain response of a single fibre-fibre interaction. This interaction is modified when fibre fragmentations are formed.

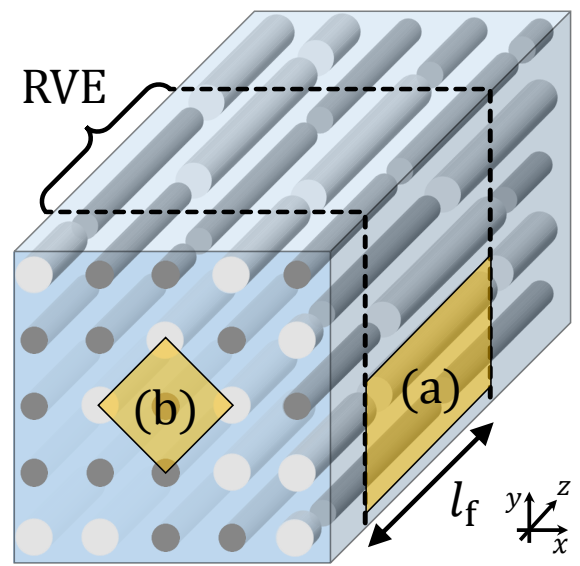

(c) All fibre stress-strain responses in a crosssection are combined in parallel to give the stress-strain response of an RVE.

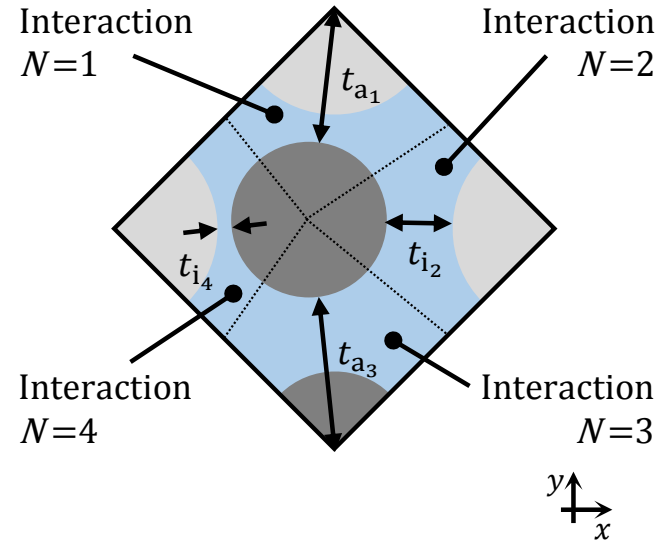

(b) The stress-strain response of the neighbouring fibre-fibre interactions are combined in parallel to give the fibre stress-strain response. The inter-fibre distance $\left(t_{\mathrm{i}_{N}}\right.$ for $N=\{2,4\})$ and fibre-centre distance $\left(t_{\mathrm{a}_{N}}\right.$ for $N=\{1,3\}$ ) are also shown.

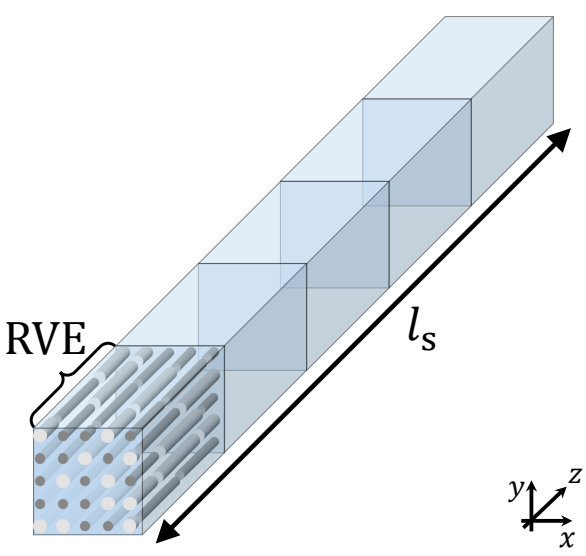

(d) All RVEs (with length $l_{\mathrm{f}}$ ) are combined in series to give the stress-strain response of the full virtual specimen (with length $l_{\mathrm{s}}$ ).

Figure 1: The stress-strain response of the ADC is calculated on multiple length scales to find the overall virtual specimen stress-strain curve (in all cases the matrix is shown in blue). 
by sampling each inter-fibre distance from a modified Poisson-Voronoi tessellation process [35]. Once the inter-fibre distances are sampled, the relative contributions from each fibre-fibre interaction to the response of a fibre are scaled according to a load-sharing coefficient (governed by a power-law); this load-sharing coefficient is calculated such that closer fibres feature a higher stress concentration, and the stress concentration carried by each fibre is inversely proportional to its structural stiffness (i.e. the product of fibre cross-sectional area and Young's modulus), regardless of the fibre-type combinations used. More details for this method can be found in Appendix A.

Table 1 shows the material properties and the values of variability parameters used for this analysis; the microstructures for each of the studied cases are also shown in Table 2 , and the influence of all sources of variability be studied in Section 3 .

\subsubsection{Modelling defects}

The VTF is also extended to model several types of defects, as follows:

- Fibre fragmentation defects (created, for example, from mishandling or damage during manufacture of the composite material) are modelled by inserting fibre fragmentations at a random location along a controlled percentage $\left(\zeta_{\mathrm{rf}}\right)$ of fibres before the start of the simulation (see Figure 2a). A fragmentation defect reduces the length of one of the two overlaps in the fibre-fibre interaction; it also creates a new broken segment in the interaction (shown with length $\delta b_{\mathrm{a}}$ in Figure 2a), whose stress-strain response is calculated in the same manner as per Henry and Pimenta 24].

- Interaction defects (created from matrix porosity or interface contamination) are simulated by setting a controlled percentage $\left(\zeta_{\mathrm{ri}}\right)$ of fibre-fibre interaction stressstrain curves to zero (thus preventing stress transfer between specific pairs of fibres), and reducing accordingly the number of active interactions $\left(N^{*}\right)$ for the fibres that neighbour the interaction defect.

- Fibre vacancy defects (created from low local fibre content and/or poor matrix consolidation) are modelled by removing fibres from the simulation; fibre removal is achieved by setting the stress-strain response of a controlled percentage of fibres $\left(\zeta_{\mathrm{fv}}\right)$ to zero, and reducing the number of active interactions accordingly for all fibres surrounding the fibre vacancy. This method effectively treats the removed 


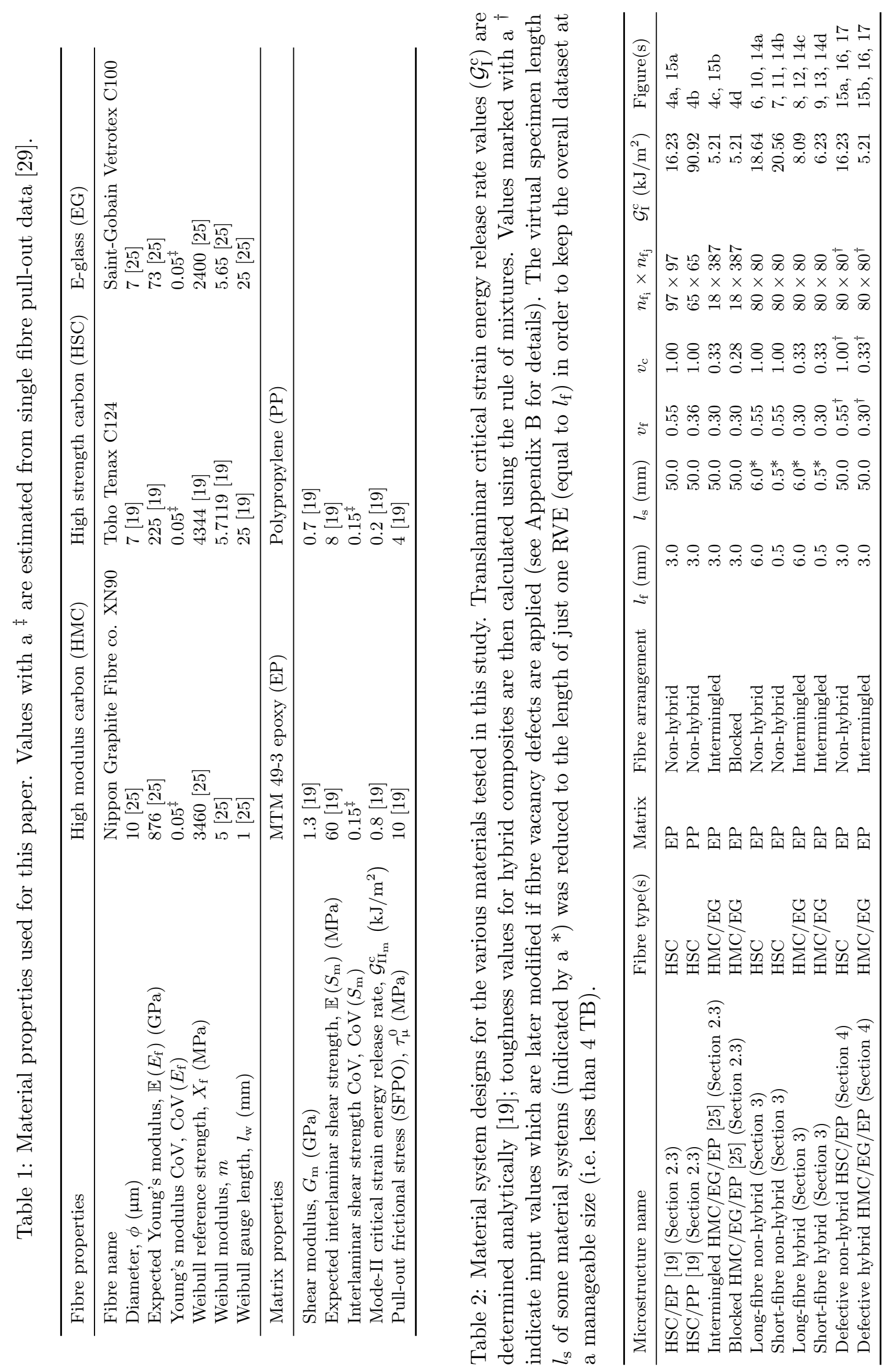




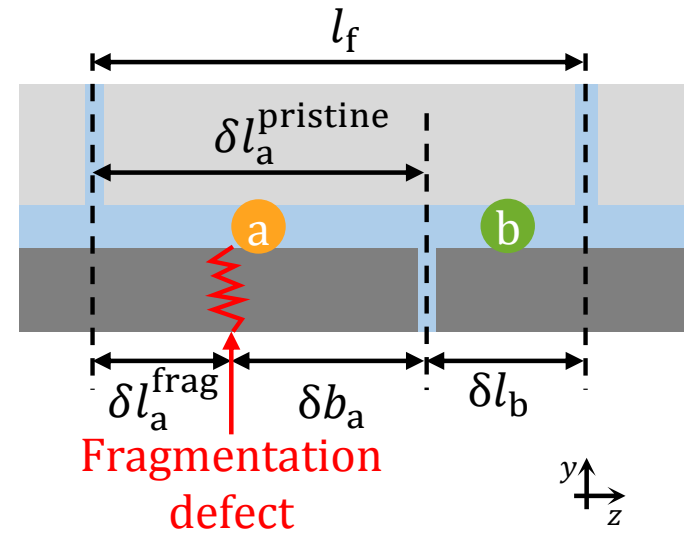

(a) Fibre fragmentation defects are created by adding a crack at a random length along the fibre. Before introduction of the fragmentation defect, the interaction is composed of overlaps $a$ and $b$ (with lengths $\delta l_{\mathrm{a}}^{\text {pristine }}$ and $\delta l_{\mathrm{b}}$, respectively); after introducing the fragmentation defect, overlap $a$ is decomposed into a shorter overlap segment (with length $\delta l_{\mathrm{a}}^{\text {frag }}$ ) and a broken segment (with length $\delta b_{\mathrm{a}}$ ).

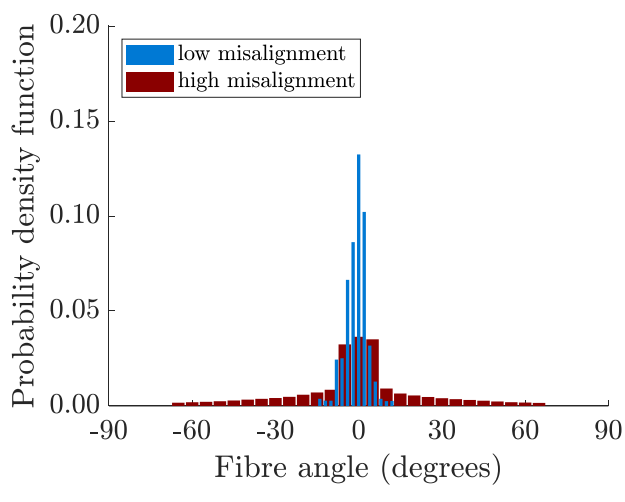

(b) High and low levels of fibre misalignment, shown as a probability density function. High levels of misalignment were recorded during work by Sanadi [38, while low levels of misalignment were measured for the HiPerDiF process [18.

Figure 2: Details for how residual fibre fragmentation and misalignment defects are modelled in the VTF.

fibres as a void within the VTF, thus simulating the presence of fibre vacancies. While removing fibres from the analysis, the fibre volume fraction (and carbon ratio for hybrids) is preserved to match the input conditions (see Appendix B for more details).

- Misalignment of the fibres (created during the manufacturing process) is simulated at the specimen-level using experimental data for the probability distribution of fibre misalignment angles (shown in Figure 2b). The loss of virtual specimen tangent stiffness due to misalignment is calculated using the equivalent laminate method [36]. Finally, Euler's method [37] is used to find the cumulative influence of misalignment on the complete stress-strain curve.

The influence of these types of defects on the mechanical performance of ADCs will be studied in Section 4

\subsubsection{Direct calculation of overlap stress-strain response}

In this work, the matrix shear response is described by a bilinear law, defined by the inter-fibre distance, matrix shear modulus, shear strength, and mode-II critical strain en- 
ergy release rate. Using a bilinear matrix constitutive law (with only two load-bearing subdomains) simplifies the shear-lag model for the stress-strain response of an overlap between two fibres significantly, when compared to the original model (which considered a matrix constitutive law with a generic number of piecewise-linear load-bearing subdomains [19, 23, 24, 25]).

This simplification makes the present implementation suitable for brittle matrices only (which are well represented by a bilinear constitutive law), such as epoxy. On the other hand, this simplification makes it possible to calculate the shear-lag response of each overlap between two fibres directly in the VTF, rather than pre-running the shear-lag model to create a library of fibre-overlap responses (as done in the previous versions of the model [19, 23, 24, 25]). This simplification is particularly important given that it would have been infeasible to create libraries of overlap responses for all combinations of stochastic fibre moduli, inter-fibre distances, and matrix properties to be considered in this work.

\subsubsection{Recording damage events}

Four different types of damage event which occur in ADCs are now identified and tracked in the VTF:

1. Matrix softening, i.e. a gradual loss of matrix shear stiffness in an interaction, leading to a progressive load reduction for a single fibre-fibre interaction, until eventually no load can be transferred across the fibre-fibre interaction.

2. Matrix debonding, caused by mode-II fracture of the interfaces in the fibre-fibre interaction, resulting in a total loss of the load-carrying capability of the interaction, and a sudden complete load drop for a single fibre-fibre interaction.

3. Fibre fragmentation, i.e. the formation of a fibre break next to a neighbouring fibreend (see Figure 1a), leading to a sudden complete load drop of a single fibre-fibre interaction, and a reduction in overlap length for all remaining neighbouring fibrefibre interactions.

4. Complete fibre failure, i.e. the inability for a fibre to receive any load from its four neighbouring fibres, due to complete matrix softening / matrix debonding / fibre fragmentation (or a mixture of these three damage events).

The VTF now records every damage event for every overlap, interaction, fibre, and RVE for each virtual specimen, along with the remote strains at which the events occurred. 
This data will be used to form damage event 'heat maps' (Section 3.1.3), in order to understand what damage events lead to final failure of the different material systems.

\subsubsection{Accurate prediction of final failure}

The full stress-strain curves of each material system described in Table 2 can be evaluated using the VTF reported above. However, earlier models [19, 25] have demonstrated the need for a fracture-mechanics-based failure criterion to truncate the full stress-strain curves of ADCs, such that the ultimate strains are predicted accurately. This is because ADCs typically fail due to the formation of clusters of damage, with one cluster in particular (from here on referred to as the critical cluster) reaching a strain energy release rate that exceeds the material critical strain energy release rate, leading to fracture of the virtual specimen. This is supported by experimental work which shows the formation of clusters of fibre breaks when loading unidirectional continuous-fibre composites [39].

Finley et al. 25] accurately predicted final failure of long-fibre intermingled and intraply hybrid ADCs by performing a fracture analysis on clusters of fully-failed fibres. However, they neglected the strain energy release from progressive matrix damage and fibre fragmentations, as well as defects, in their fracture analysis. Finley's approach [25] works well for cases where failure is dominated by fibre failure (such as in long-fibre hybrid ADCs), but it cannot be applied to cases where sub-critical damage (e.g. matrix damage and/or fibre fragmentations that do not lead to complete fibre failure) or defects may contribute significantly to final failure. In this section, a new fracture criterion will be developed, which considers not only fibre failure but also other forms of damage (including contributions from progressive matrix softening / debonding, sub-critical fibre fragmentations, and defects), in order to achieve an accurate prediction of final failure in any type of aligned discontinuous composite.

The new fracture criterion follows the flowchart in Figure 3. Firstly, several properties are loaded into the VTF, including material properties (see Table 1), the number of fibres in the $x$ - and $y$-dimensions of the cross-section $\left(n_{\mathrm{f}_{\mathrm{j}}}\right.$ and $n_{\mathrm{f}_{\mathrm{i}}}$ respectively), diameters of the carbon and glass fibres $\left(\phi_{\mathrm{c}}\right.$ and $\left.\phi_{\mathrm{g}}\right)$, matrix of fibre types $\boldsymbol{T}_{\mathrm{f}}$, matrix of fibre cross-sectional areas $\boldsymbol{A}_{\mathrm{f}}$, fibre volume fraction $v_{\mathrm{f}}$, composite translaminar critical strain energy release rate $\mathcal{G}_{\mathrm{I}}^{\mathrm{c}}$ (calculated analytically, as mentioned in Table 2), and the strain increment $\Delta \varepsilon$ (Step I.1, Figure 3). Throughout the analysis, the strain index $v$ is used to determine the applied remote strain $\varepsilon^{\infty[v]} ; r$ and $k$ are also used throughout the analysis to denote the RVE index and the cluster index, while $i$ and $j$ denote the $y$ and $x$ cross-sectional 
locations of each fibre, respectively. Next, the stress-strain curves of all fibres (stored in the $\boldsymbol{\sigma}_{\mathrm{f}}^{[i, j, r, v]}$ matrix and the $\boldsymbol{\varepsilon}^{\infty[v]}$ vector), and of all RVEs (stored in the $\boldsymbol{\sigma}_{\mathrm{RVE}}^{[r, v]}$ matrix and $\varepsilon^{\infty[v]}$ vector) of the un-fractured virtual specimen are calculated using the VTF described in Sections 2.1 to 2.2 (Step I.2, Figure 3).

Once the un-fractured stress-strain curves are determined, the point of fracture can then be determined (steps II to V in Figure 3), as explained hereafter. Starting from a remote strain of zero (step II), the damage state $\boldsymbol{D}_{\mathrm{f}}$ of every fibre is quantified as a loss of equivalent fibre stiffness, according to

$$
\boldsymbol{D}_{\mathrm{f}}^{[i, j, r, v]}=1-\frac{\boldsymbol{\sigma}_{\mathrm{f}}^{[i, j, r, v]}}{\boldsymbol{E}_{\mathrm{f}}^{[i, j, r]} \cdot \boldsymbol{\varepsilon}^{\infty[v]}},
$$

where $\boldsymbol{\sigma}_{\mathrm{f}}^{[i, j, r, v]}$ is the fibre stress at the given $i$ and $j$ locations in the $r^{\text {th }}$ RVE at the strain index $v$, and $\boldsymbol{E}_{\mathrm{f}}^{[i, j, r]}$ is the equivalent initial fibre stiffness. A value of $\boldsymbol{D}_{\mathrm{f}}^{[i, j, r, v]}=1$ means the fibre in question is fully damaged (and thus cannot carry any load), whereas a value of $\boldsymbol{D}_{\mathrm{f}}^{[i, j, r, v]}=0$ means the fibre is un-damaged. Next, a damage threshold $d$ (the amount of damage that a fibre may have before it is considered to be part of a cluster) is selected (step III.1); any fibres with a damage state that is greater than or equal to $d$ are considered part of a damaged cluster (step III.2). Once the clusters are defined, the cluster opening stress $\boldsymbol{\sigma}_{\mathrm{o}}^{[r, v, d]}$ is calculated for the $r^{\text {th }}$ RVE as the remote stress minus the contribution of any residual stresses carried by all damaged clusters in that RVE (step III.5). The strain energy release rate $\boldsymbol{J}^{[k, r, v, d]}$ associated with fracture propagating from each damaged cluster is then calculated according to Dugdale's strip-yield model [19] (step IV.2), assuming an equivalent-sized penny-shaped crack of radius $\boldsymbol{a}_{\mathrm{e}}^{[k, r, v, d]}$ which is scaled by the damage state in the fibres.

Steps III.1 to IV.2 are repeated for every damage threshold $d$ from 1 to 0 , as shown in Figure 3. The minimum fracture reserve factor $\boldsymbol{R}_{\min }^{[r, v]}$, i.e. the minimum ratio of the composite translaminar critical strain energy release rate $\mathcal{G}_{\mathrm{I}}^{\mathrm{c}}$ and the strain energy release rate $\boldsymbol{J}^{[k, r, v, d]}$, is then calculated (step V.1). If the minimum reserve factor is less than 1 , the material has fractured at the current remote strain $\varepsilon^{\infty[v]}$; otherwise, the strain index $v$ is increased and steps II to V.1 are repeated again until fracture is detected. This whole process is then repeated for every RVE within the virtual specimen; the virtual specimen stress-strain curve is subsequently trimmed to the fracture strain index $\widetilde{v}$, i.e. the minimum strain index at which fracture occurs in any of the RVEs. 


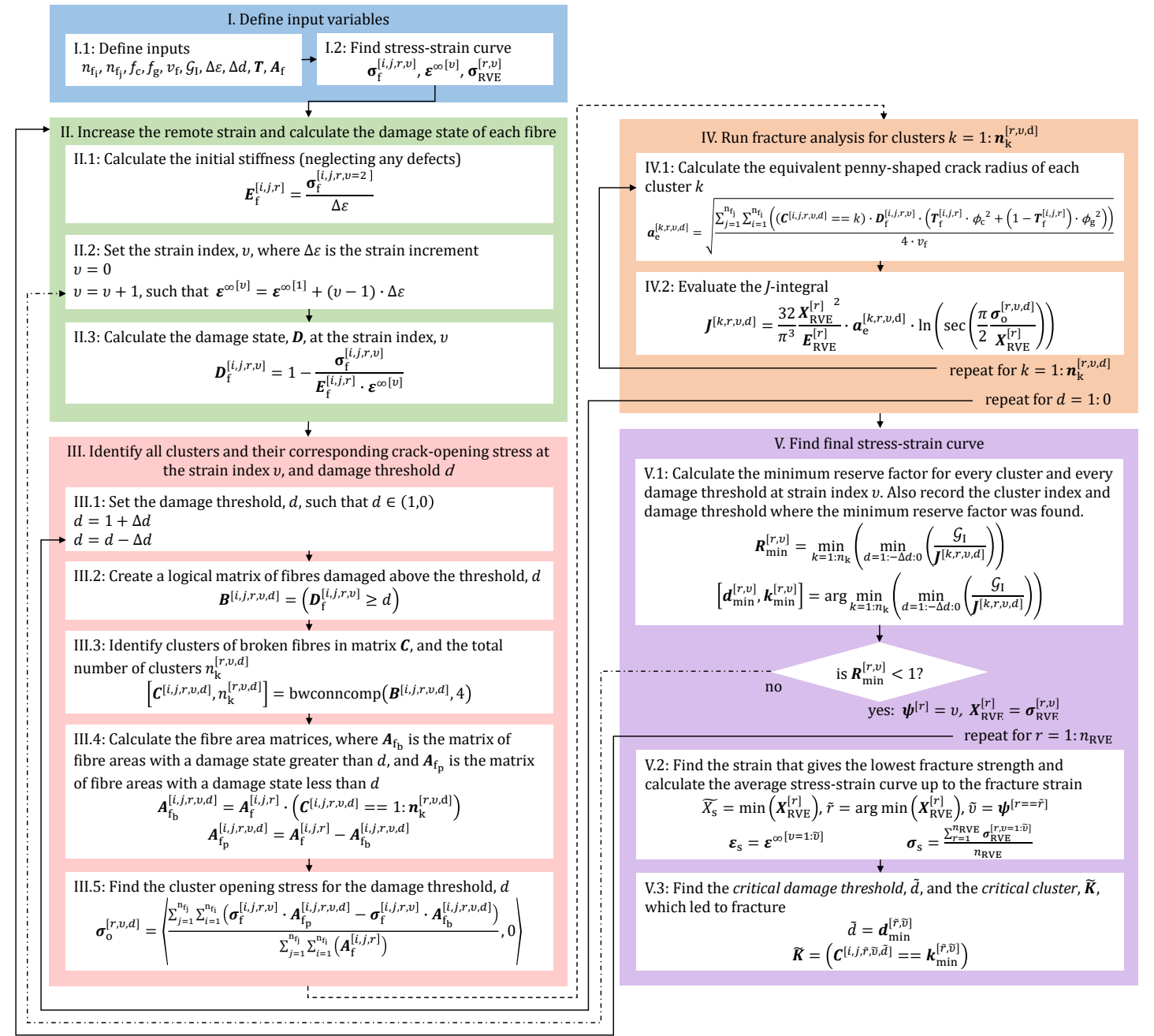

Figure 3: Implementation of the fracture-based failure criterion. ' $==$ ' denotes the logical operation 'if equal to', arg $\min ()$ stands for 'argument(s) of the minimum', and 'bwconncomp' is a MATLAB function used to identify unique clusters within an array [40].

The critical cluster $\widetilde{\boldsymbol{K}}$ is defined as the cluster of damage which led to fracture of the virtual specimen; the critical cluster also defines the most critical RVE index $\widetilde{r}$, at the fracture strain $\varepsilon^{\infty[\tilde{v}]}$, and at the most critical damage threshold $\widetilde{d}$. The size, shape, and location of the critical cluster are recorded for future analysis (Sections 3.1, and 4.1). If fracture is never predicted to occur using the strain energy release rate criterion in Step V.1 (Figure 3), the virtual specimen fails when its ultimate strength is reached; in this case the critical cluster is not recorded and a flag is used to record a strength-based failure.

\subsection{Validation against experiments}

The VTF was used with the new features described in Section 2.2 to predict the mechanical response of four very different types of ADC: HSC/EP (high strength car- 
bon fibre / epoxy), HSC/PP (high strength carbon fibre / polypropylene), intermingled HMC/EG/EP (high modulus carbon fibre / E-glass / epoxy with an intermingled fibretype arrangement), and intraply blocked HMC/EG/EP (the same constituents as the intermingled hybrid, but with an intraply fibre-type arrangement - see previous work by Finley et al. 25] for more details on the intraply blocked HMC/EG/EP material system); the material properties and microstructural designs of these validation cases are specified in Table 1 and Table 2 respectively. Each plot in Figure 4 shows the stress-strain curve of one representative virtual specimen, and the fracture points from three different virtual specimens for each material system. The VTF predictions show good agreement with experimental data for all types of ADC (as shown in Figure 4), for both a strong (epoxy) and a weak (polypropylene) matrix (Figures $4 \mathrm{a}$ and $4 \mathrm{~b}$ respectively). There is also good agreement with hybrid ADCs, either with an intermingled fibre-type arrangement (Figure $4 \mathrm{c}$ ), or with an intraply fibre-type arrangement featuring a single large block of carbon fibres (Figure $4 \mathrm{~d}$ ). 


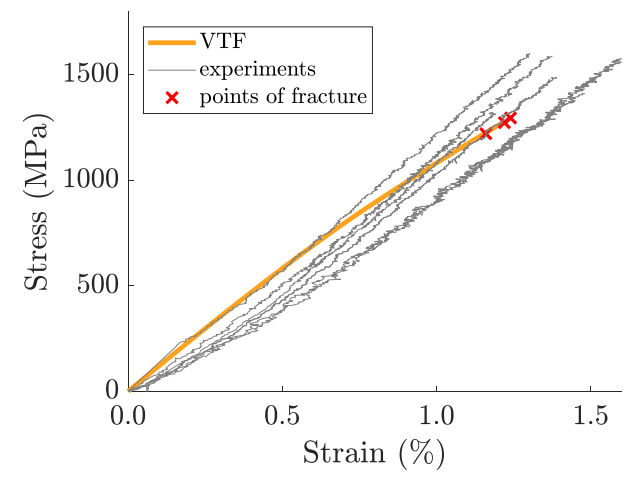

(a) HSC/EP ADCs (experimental data from Yu et al. [18]).

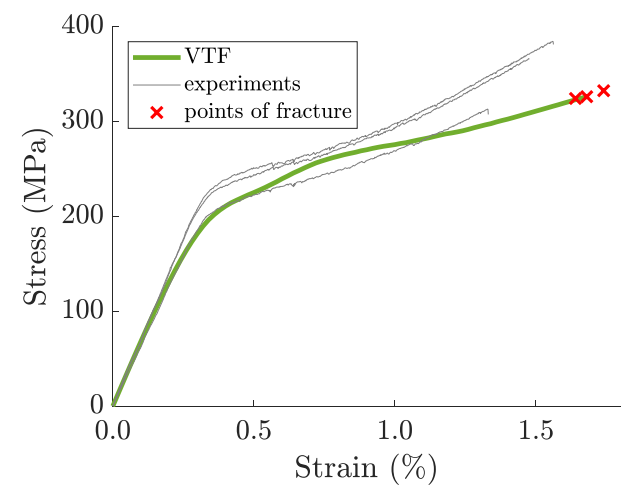

(c) Intermingled $\mathrm{HMC} / \mathrm{EG} / \mathrm{EP}$ hybrid ADCs (experimental data from Finley et al. [25]).

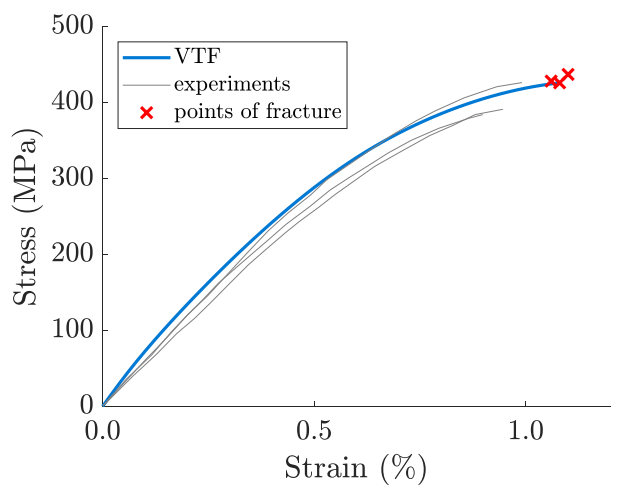

(b) HSC/PP ADCs (experimental data from Yu et al. 22]).

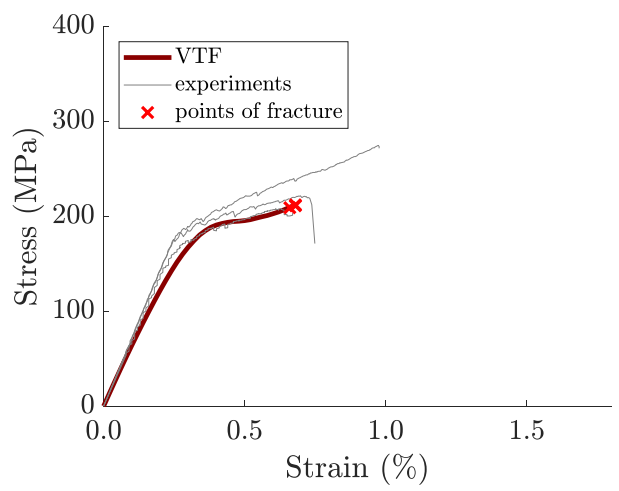

(d) Blocked HMC/EG/EP intraply hybrid ADCs (experimental data from Finley et al. [25]).

Figure 4: A comparison between experimental data and VTF predictions. The VTF stressstrain curves varied very little between repeat simulations, with only the final fracture point varying significantly, hence only one VTF stress-strain curve is shown per virtual specimen. The VTF shows good agreement with experiments for a variety of aligned discontinuous composite material systems. 


\section{The influence of variability on mechanical performance}

This section investigates the influence of variability on the tensile response of four different ADC material systems: long-fibre non-hybrid, short-fibre non-hybrid, long-fibre hybrid, and short-fibre hybrid; the material properties and microstructural designs are specified in Table 1 and Table 2 respectively. In order to generate statistically relevant results, 4000 virtual tests were completed for each material system; the critical cluster location and material properties were recorded for each virtual specimen, so that the properties of the critical cluster could be compared against those of the bulk composite.

\subsection{Results}

\subsubsection{Material property 'heat maps'}

Using the method summarised in Figure 5 (which is similar to that by de Geus et al. [41]), material property 'heat maps' were generated for long-fibre ADCs (Figure 6), short-fibre ADCs (Figure 7), long-fibre hybrid ADCs (Figure 8), and short-fibre hybrid ADCs (Figure 9). These heat maps enable the comparison between the properties of

2. Next, a non-zero value $\rho$ is added to the canvas in the same $i$ and $j$ fibre locations as where the critical cluster from the first virtual specimen is found (using the critical cluster matrix $\widetilde{\boldsymbol{K}}$ from Section 2.2.5). This non-zero value $\rho$ is calculated from the number of fibres in the critical cluster $\left(n_{\mathrm{f}_{\widetilde{\mathrm{k}}}}\right)$ as

$$
\rho=\frac{1}{n_{\mathrm{f}_{\tilde{\mathrm{k}}}}} .
$$




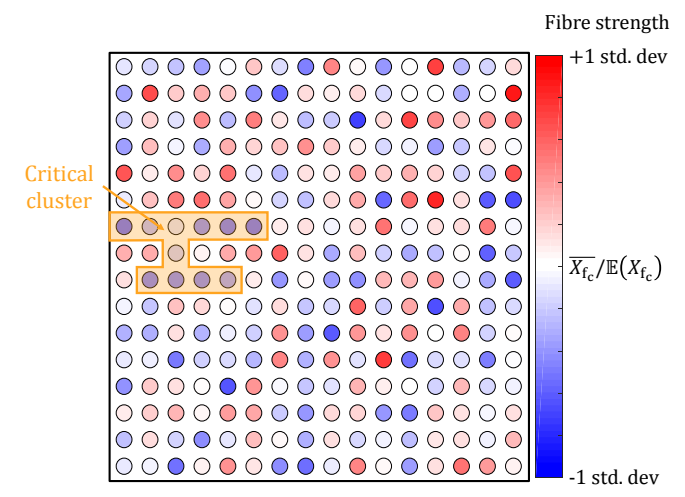

(a) The VTF is used to determine the location of the critical cluster in the virtual specimen cross-section; the virtual specimen properties (normalised fibre strength in this case) are also recorded. 4000 virtual tests were run to create a statistically representative collection of virtual specimens and critical clusters.

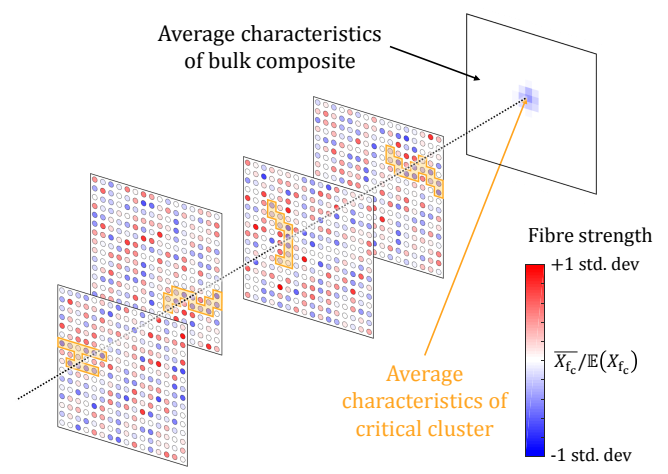

(b) All of the virtual specimens are concatenated on top of one-another, and each of the virtual specimens is translated such that the centroid of all critical clusters lie on the same axis. The local properties of the virtual specimens are then averaged along the alignment axis to give the properties of the critical cluster (close to the alignment axis) and the bulk composite (elsewhere).

Figure 5: Using a method first proposed by de Geus et al. [41], material property 'heat maps' can be created, so that the relative properties of the critical cluster can be compared to those of the bulk composite. This specific example demonstrates that the fibres in the critical cluster are, on average, weaker than those in the bulk composite (for this particular material system).

3. Step 2 is repeated for every virtual specimen, and the whole canvas is then divided by the number of virtual specimens to give a normalised critical cluster location map.

Normalised critical cluster location maps were produced for long-fibre ADCs (Figure 6a), short-fibre ADCs (Figure 7a), long-fibre hybrid ADCs (Figure 8a), and short-fibre hybrid ADCs (Figure 9a).

\subsubsection{Damage event 'heat maps'}

The same 'heat map' technique as described in Section 3.1.1 was used to record the relative proportion of different damage events (as defined in Section 2.2.4 ) that occurred within the critical cluster of different composites, in order to see what forms of damage contribute to fracture of the different material systems. The damage analysis was performed for all four composite types, the results of which are shown in Figures 10 to 13 .

\subsubsection{Critical damage threshold}

During the fracture analysis, the critical damage threshold $(\widetilde{d}$, i.e. the damage threshold at which the critical cluster caused fracture of the virtual specimen - see Section 2.2.5 for 


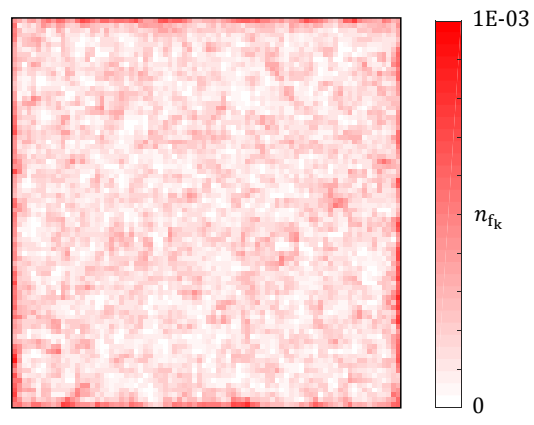

(a) Normalised critical cluster location map; see Section 3.1 .2 for details.
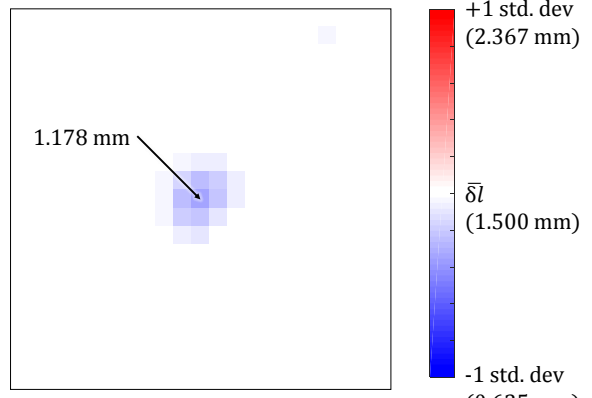

$(0.635 \mathrm{~mm})$

(c) Heat map for fibre overlap length variability.

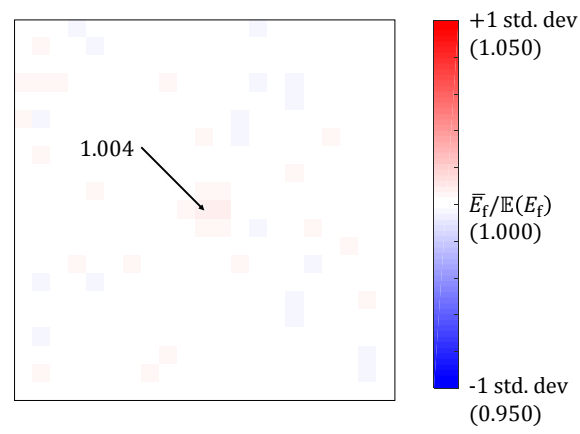

(e) Heat map for fibre modulus variability (normalised to the expected fibre modulus).

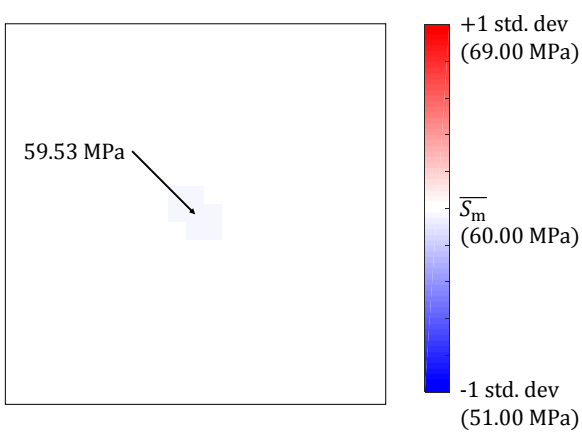

(b) Heat map for matrix strength variability.

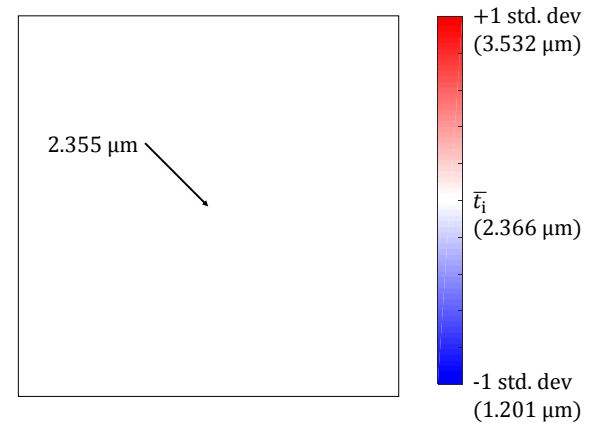

(d) Heat map for inter-fibre distance variability.

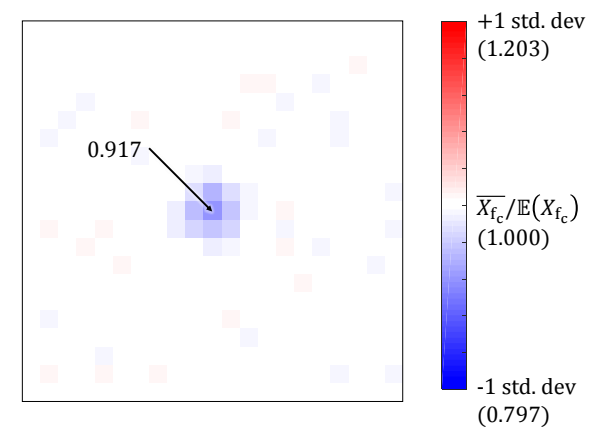

(f) Heat map for carbon fibre strength variability (normalised to the expected fibre strength).

Figure 6: Heat maps for the long-fibre non-hybrid ADC material system. The normalised critical cluster location map is shown in Figure 6a, while heat maps for sources of microstructure variability are shown on zoomed-in canvases (forming 21 by 21 fibre regions) in Figures 6b to 6f 


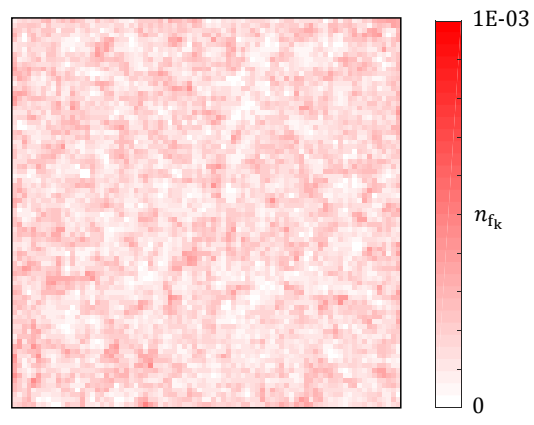

(a) Normalised critical cluster location map; see Section 3.1 .2 for details.
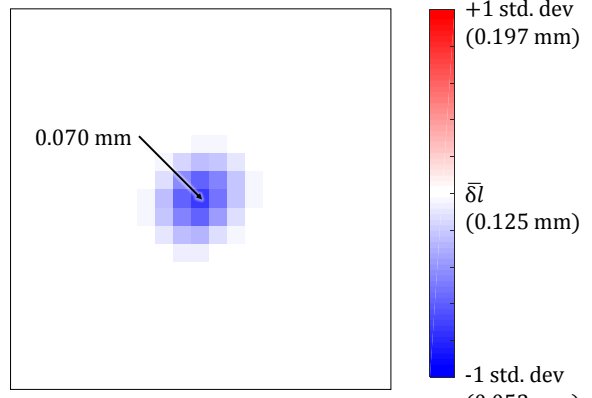

(c) Heat map for fibre overlap length variability.

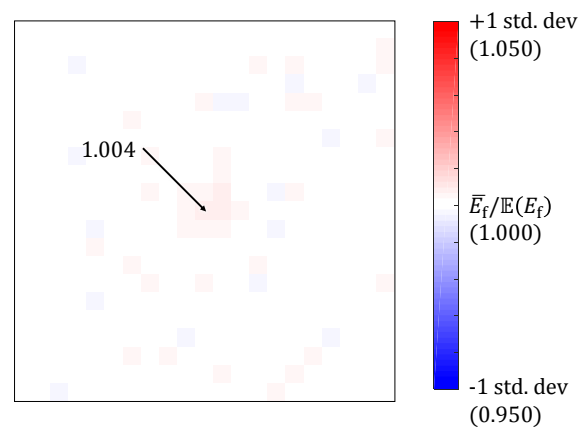

(e) Heat map for fibre modulus variability (normalised to the expected fibre modulus).

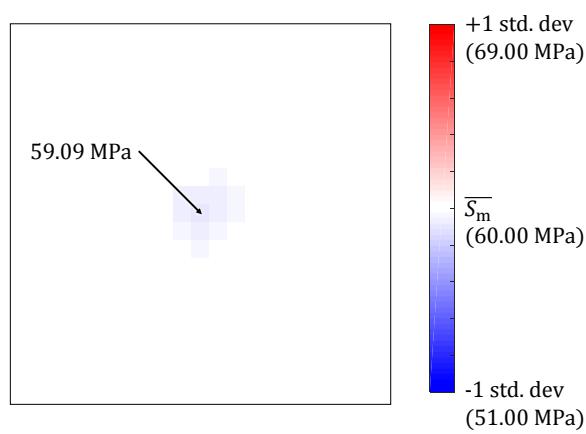

(b) Heat map for matrix strength variability.

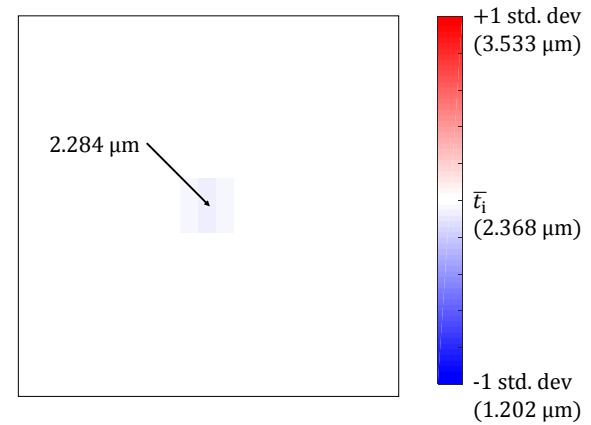

(d) Heat map for inter-fibre distance variability.

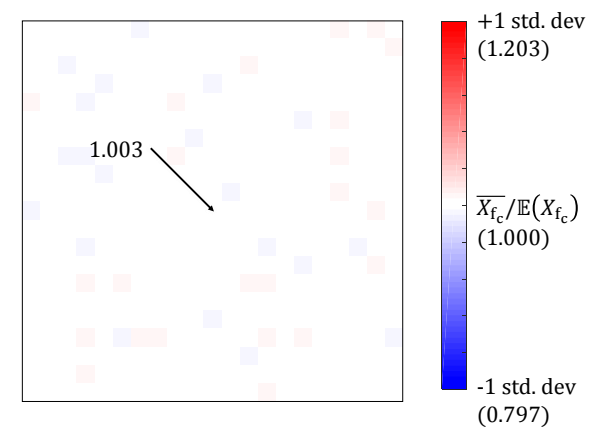

(f) Heat map for carbon fibre strength variability (normalised to the expected fibre strength).

Figure 7: Heat maps for the short-fibre non-hybrid ADC material system. The normalised critical cluster location map is shown in Figure 7a, while heat maps for sources of microstructure variability are shown on zoomed-in canvases (forming 21 by 21 fibre regions) in Figures $7 \mathrm{~b}$ to $7 \mathrm{f}$. 


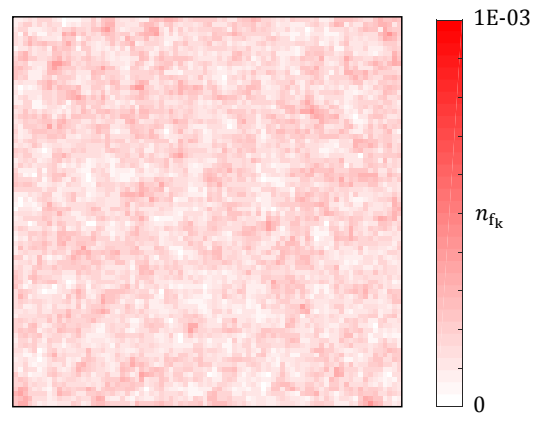

(a) Normalised critical cluster location map; see Section 3.1 .2 for details.
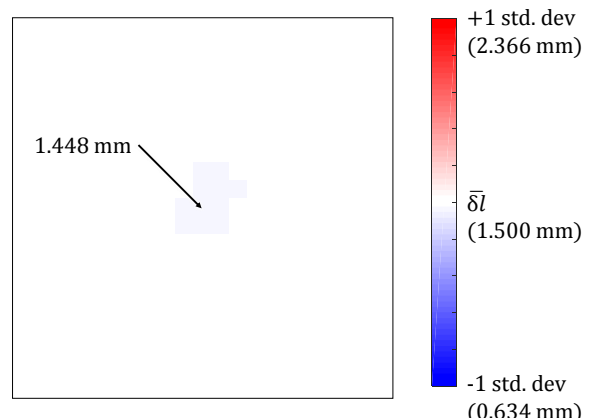

(c) Heat map for fibre overlap length variability.

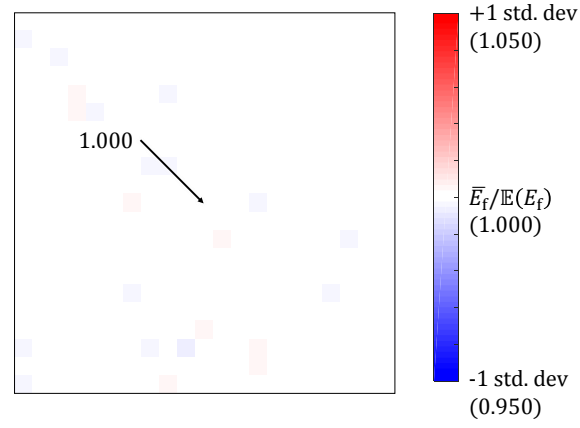

(e) Heat map for fibre modulus variability (normalised to the expected fibre modulus).

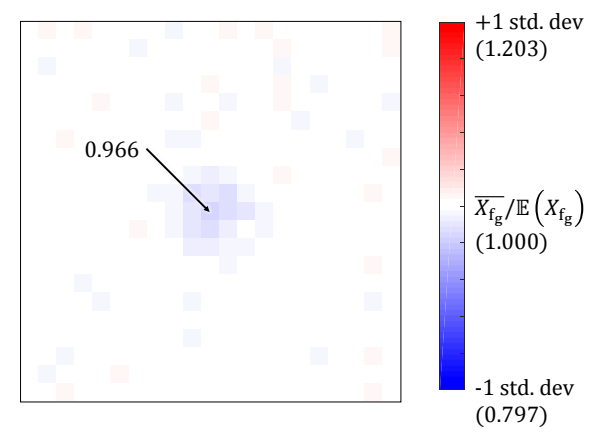

(g) Heat map for glass fibre strength variability (normalised to the expected glass fibre strength).

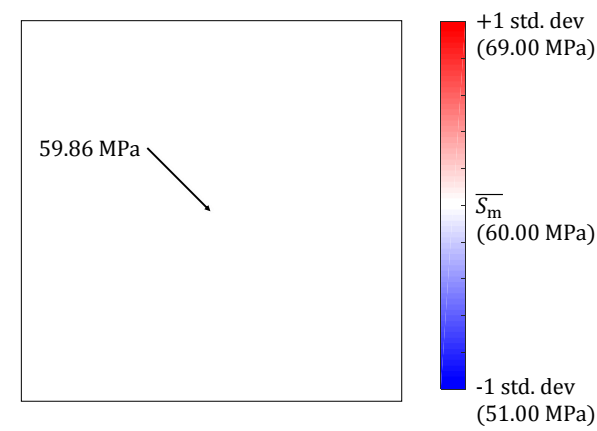

(b) Heat map for matrix strength variability.

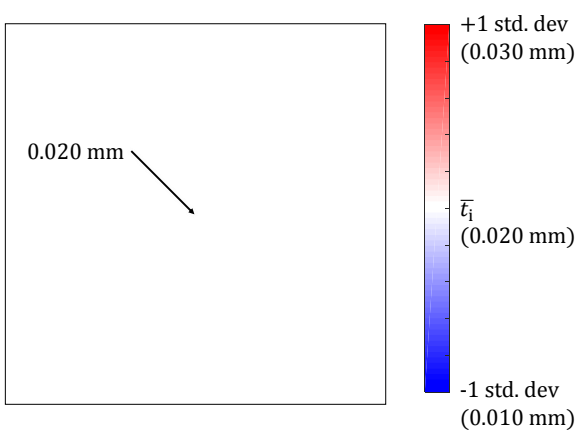

(d) Heat map for inter-fibre distance variability.

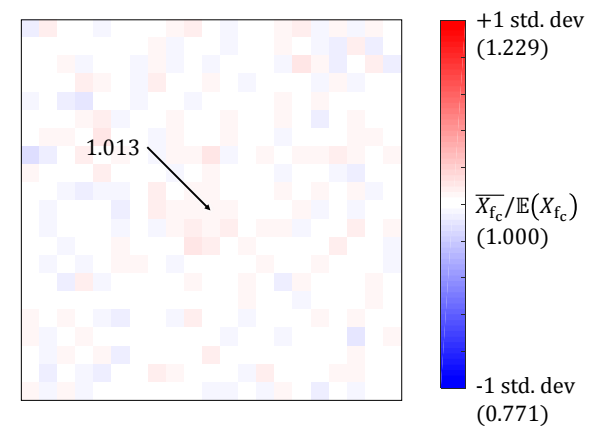

(f) Heat map for carbon fibre strength variability (normalised to the expected carbon fibre strength).

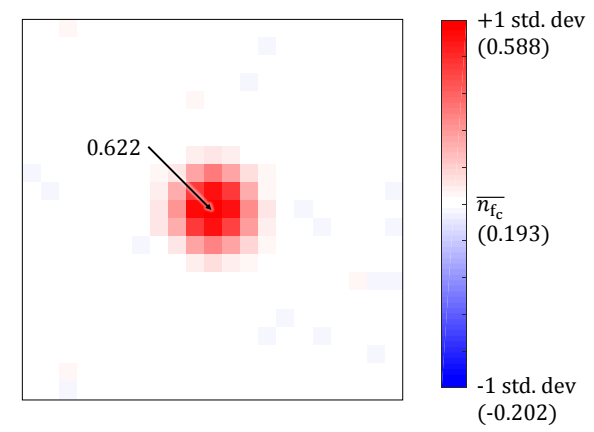

(h) Heat map for the local number fraction of low-elongation fibres.

Figure 8: Heat maps for the long-fibre hybrid ADC material system. The normalised critical cluster location map is shown in Figure 8a, while heat maps for sources of microstructure variability are shown on zoomed-in canvases (forming 21 by 21 fibre regions) in Figures 8b to 8h. 


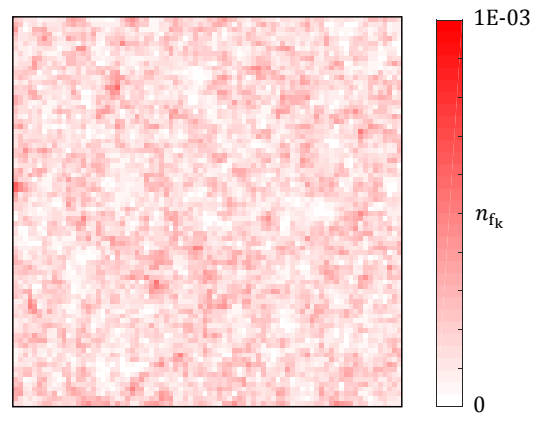

(a) Normalised critical cluster location map; see Section 3.1 .2 for details.
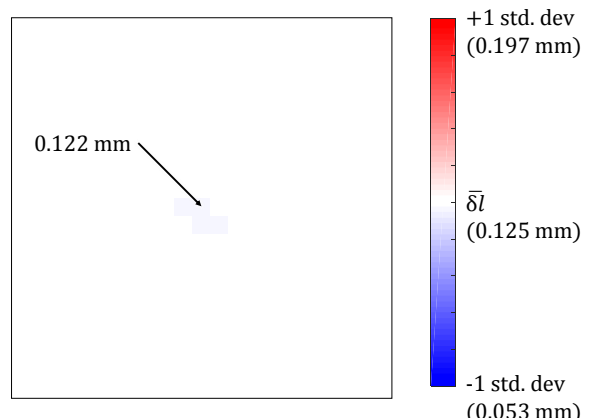

(c) Heat map for fibre overlap length variability.

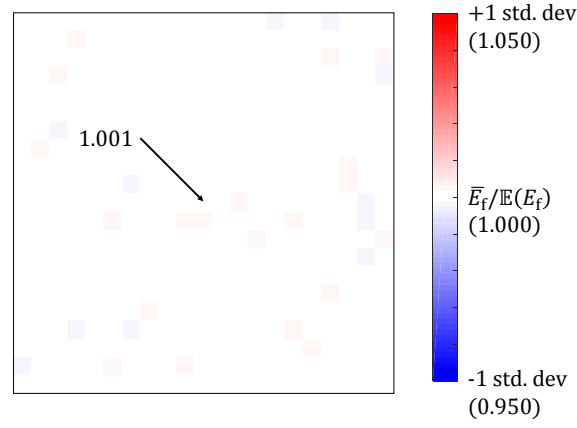

(e) Heat map for fibre modulus variability (normalised to the expected fibre modulus).
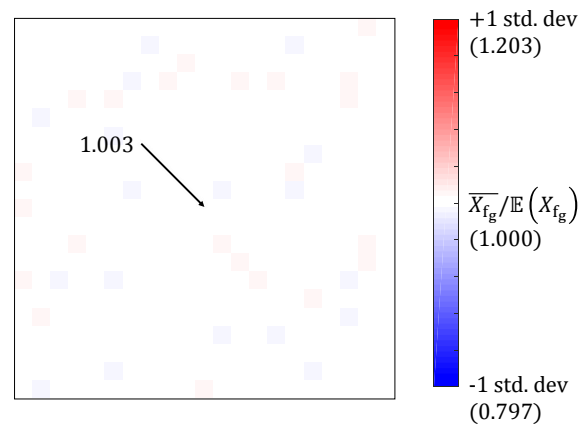

(g) Heat map for glass fibre strength variability (normalised to the expected glass fibre strength).

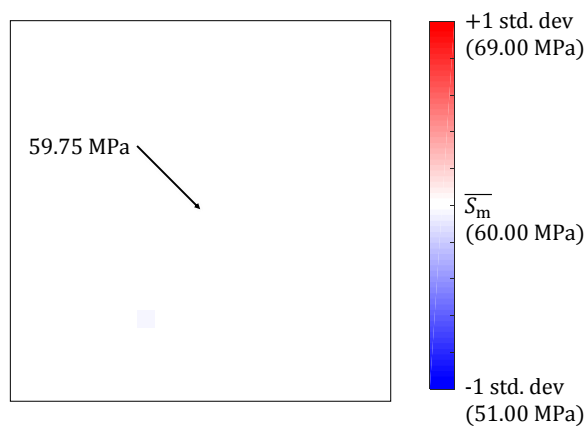

(b) Heat map for matrix strength variability.

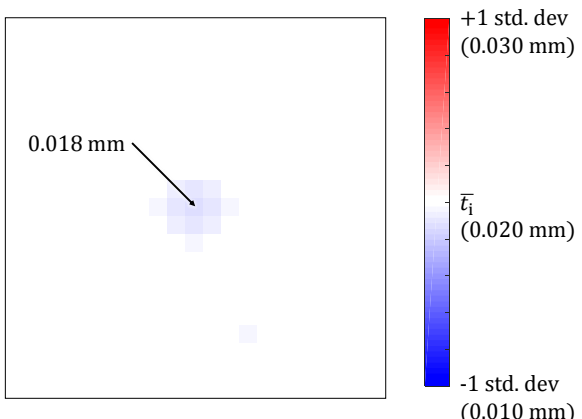

(d) Heat map for inter-fibre distance variability.

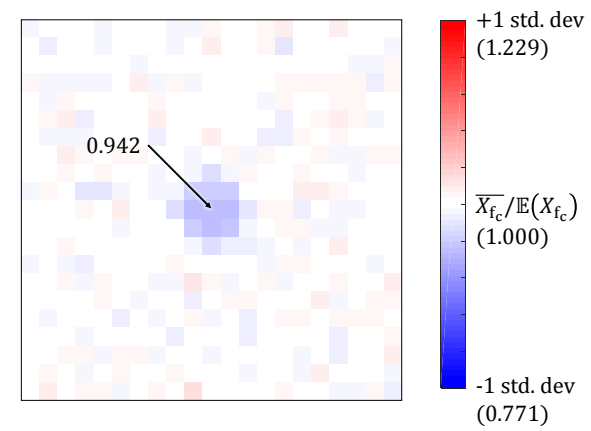

(f) Heat map for carbon fibre strength variability (normalised to the expected carbon fibre strength).

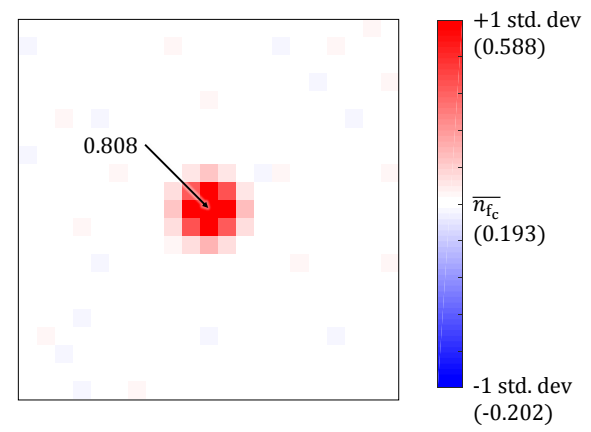

(h) Heat map for the local number fraction of low-elongation fibres.

Figure 9: Heat maps for the short-fibre hybrid ADC material system. The normalised critical cluster location map is shown in Figure 9a, while heat maps for sources of microstructure variability are shown on zoomed-in canvases (forming 21 by 21 fibre regions) in Figures $9 \mathrm{~b}$ to $9 \mathrm{~h}$. 


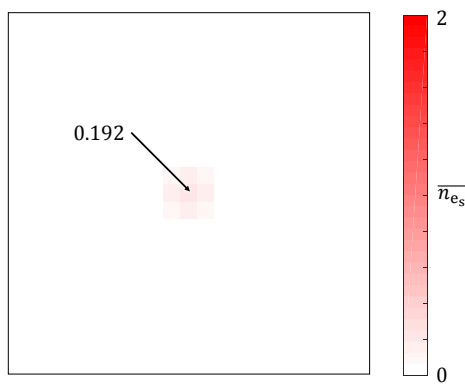

(a) Average number of matrix softening events.

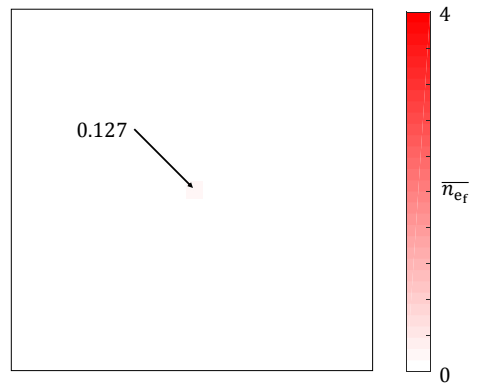

(c) Average number of fibre fragmentation events.

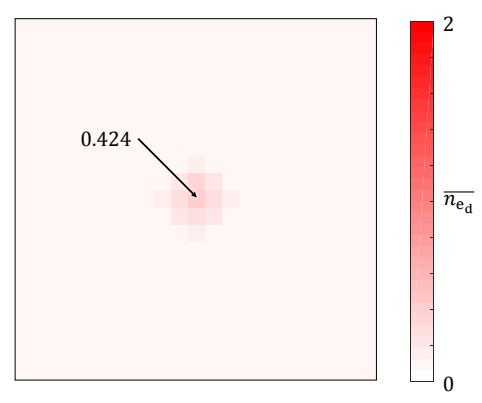

(b) Average number of debonding events.

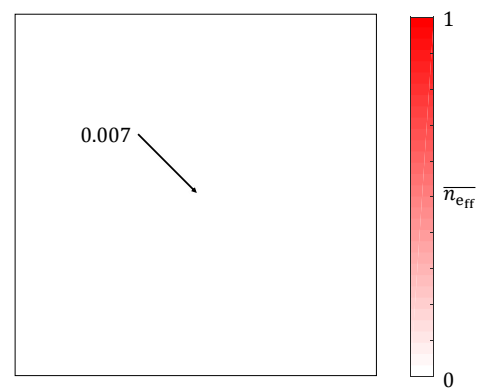

(d) Average number of fibre failure events (i.e. when the fibre can no longer carry load).

Figure 10: Damage event heat maps for the long-fibre non-hybrid ADC material system. Debonding is the critical source of damage within long-fibre non-hybrid ADCs.

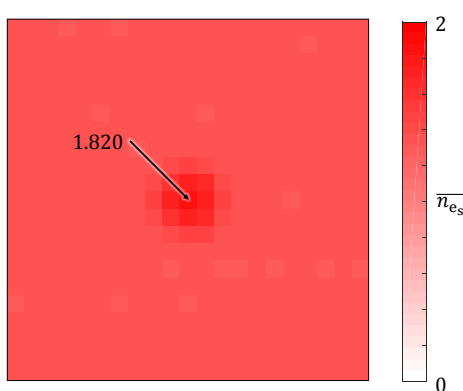

(a) Average number of matrix softening events.

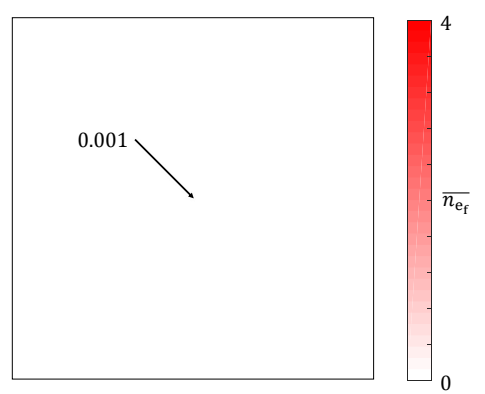

(c) Average number of fibre fragmentation events.

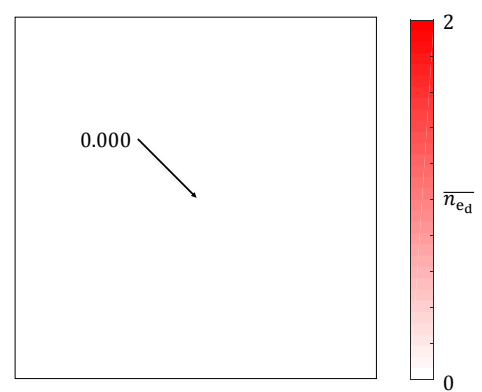

(b) Average number of debonding events.

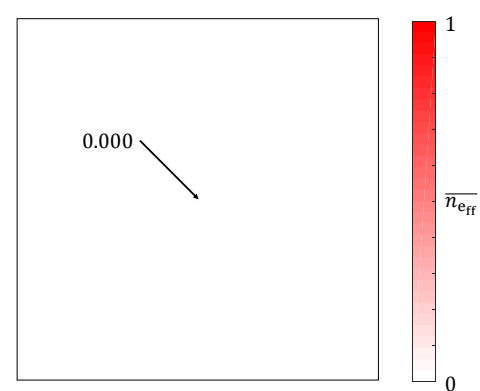

(d) Average number of fibre failure events.

Figure 11: Damage event heat maps for the short-fibre non-hybrid ADC material system. Matrix softening is the critical source of damage within short-fibre non-hybrid ADCs. 


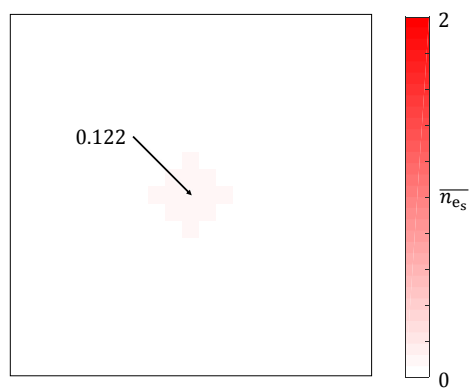

(a) Average number of matrix softening events.

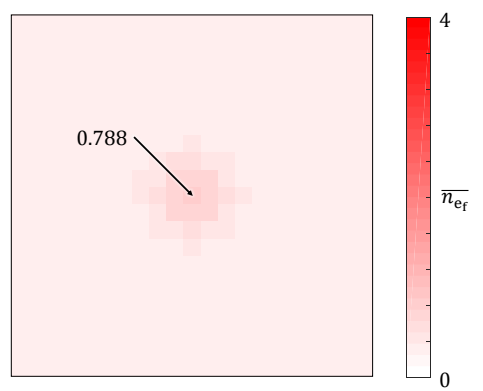

(c) Average number of fibre fragmentation events.

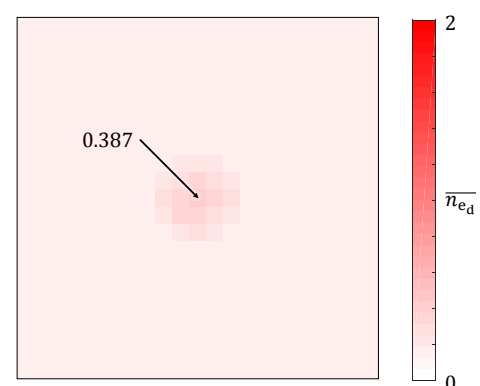

(b) Average number of debonding events.

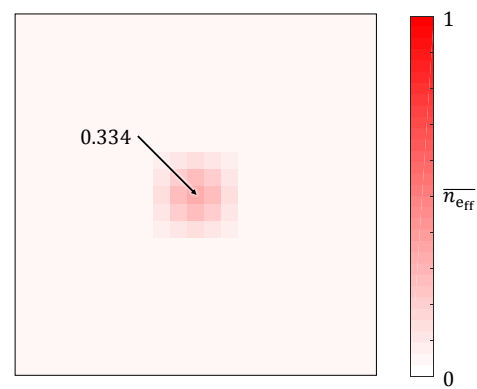

(d) Average number of fibre failure events.

Figure 12: Damage event heat maps for the long-fibre hybrid ADC material system. Many fibre fragmentations and debonding events led to a large number of fibre failure events within long-fibre hybrid ADCs. 


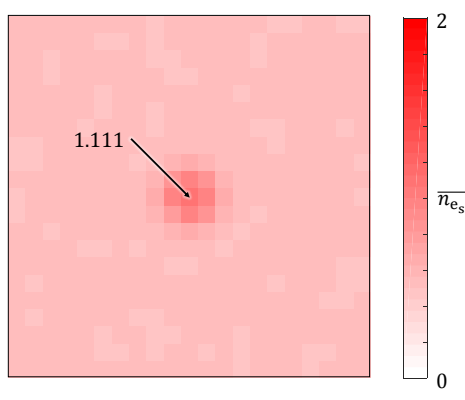

(a) Average number of matrix softening events.

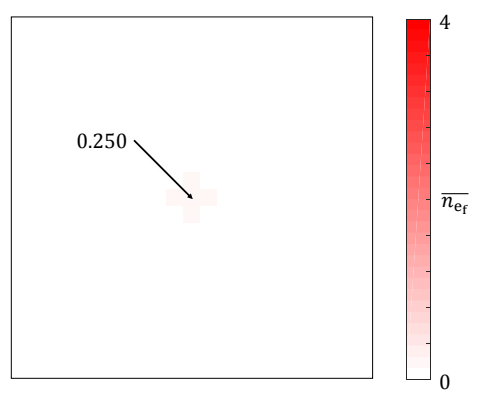

(c) Average number of fibre fragmentation events.

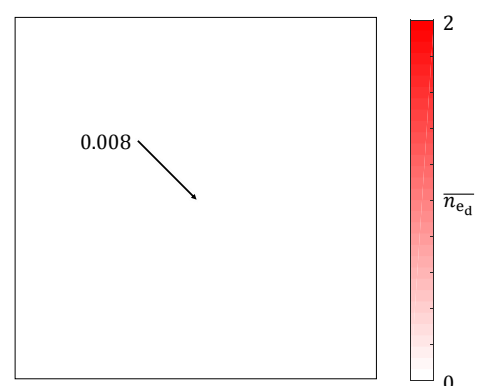

(b) Average number of debonding events.

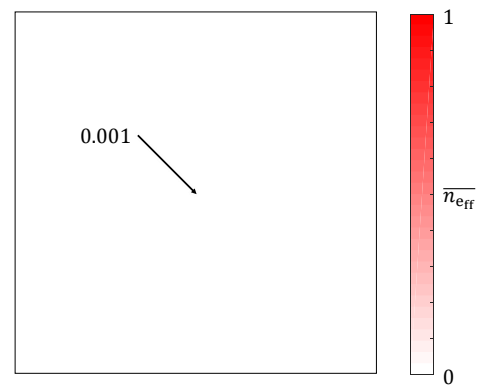

(d) Average number of fibre failure events.

Figure 13: Damage event heat maps for the short-fibre hybrid ADC material system. Matrix softening and fibre fragmentation are the critical sources of damage within shortfibre hybrid ADCs. 


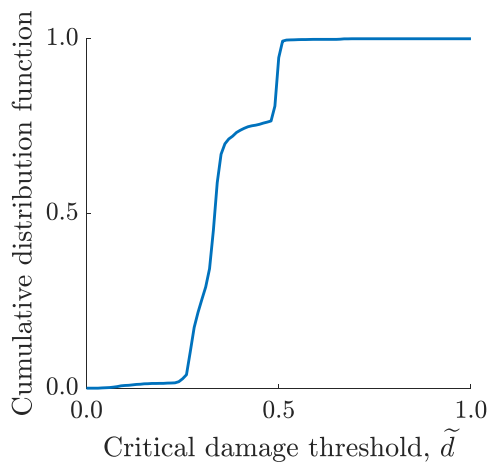

(a) Long-fibre non-hybrid ADCs.

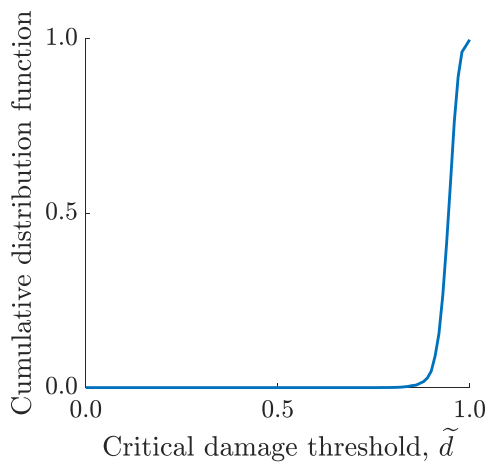

(c) Long-fibre hybrid ADCs.

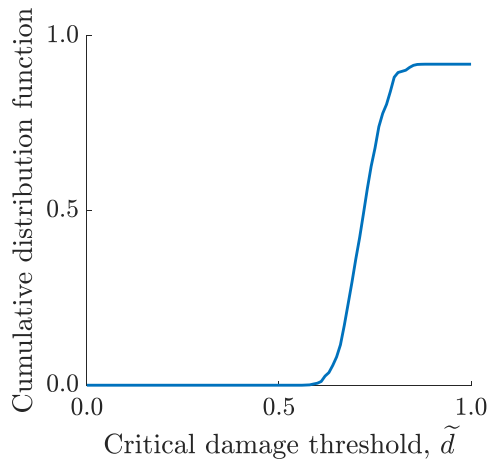

(b) Short-fibre non-hybrid ADCs.

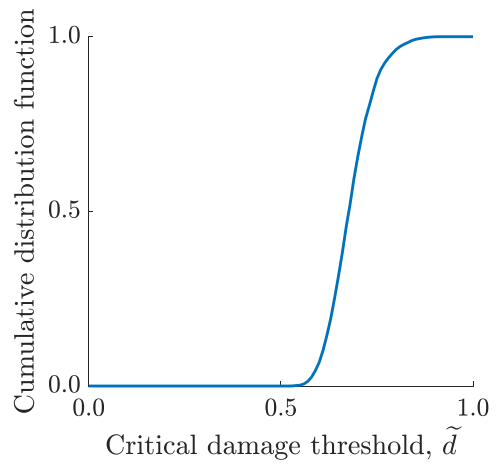

(d) Short-fibre hybrid ADCs.

Figure 14: The critical damage threshold follows a different distribution, depending on the composite type.

details) was recorded for every virtual specimen. Cumulative distribution function (CDF) plots were then generated to show the distribution of critical damage threshold for each material system, as shown in Figure 14.

\subsection{Discussion}

The results from Section 3.1 are summarised in Table 3. The critical source of variability (i.e. the source of variability which led to final failure) depends on the material system, as explained below:

- In general, non-hybrid ADCs are sensitive to variability in the overlap length (see Figures 6c and 7c), as shorter overlaps lead to matrix softening and/or debonding of the matrix; this is consistent with the predominance of softening and debonding damage events that were recorded for these material systems (see Figures 10a, 10b, and 11a).

- The long-fibre non-hybrid material system is more sensitive to variability in the fibre strength (see Figures 6f and 10d) than its short-fibre counterpart; this sensitivity is 
because the longer overlaps in the long-fibre non-hybrid material system are able to generate sufficient stresses to fragment the fibres.

- The hybrid ADCs are dominated by the variability in local fibre-type arrangement (see Figures $8 \mathrm{~h}$ and 9h), as large groups of low-elongation (brittle high-modulus carbon) fibres promote stress concentrations that lead to matrix damage (i.e. debonding and matrix softening) and fibre fragmentation (see Figures 12a, 12b, 12c, and 13a).

- The long-fibre hybrid ADC system undergoes a significant amount of debonding and fragmentation damage events during loading, causing dispersed fibres to fully fail before final failure (Figure 12d).

Next, the dominant source of ductility within each material system can be determined. Ductility can be investigated by observing whether a given type of damage event is present away from the critical cluster region of the damage event heat maps (Figures 10 to 13 ), as this implies that this damage event is diffuse within the bulk of the material (and hence contributes to a significant amount of inelastic deformation). An example of ductile damage can be seen in the matrix softening damage event heat map for the short-fibre hybrid ADC (Figure 13a); on the contrary, fibre fragmentation events are only present in the critical cluster, and hence promote final failure without any additional ductility (see Figure 13c). The source(s) of ductility are summarised for each material system in Table 3 ,

Finally, it is clear from Figure 14 that the critical damage threshold distributions are different for each material system, with each plot offering additional insight into the type(s) and amounts of damage event(s) that led to fracture in each case:

- As described in Section 2.2.5, a damage threshold of 1 implies that fibres within a cluster are fully damaged and are unable to carry any load; this is observed in the long-fibre hybrid ADC material system, where the critical damage threshold is often around 1, which is consistent with the large number of fibre failure events that were recorded (see Figures $14 \mathrm{c}$ and $12 \mathrm{~d}$ ).

- The long-fibre non-hybrid ADC features both debonding and fibre fragmentations (see Figures $10 \mathrm{~b}$ and 10c, which both work to cause one of the four fibre-fibre interactions to fail. Failure of an interaction reduces the stiffness of the fibre stressstrain curve by around $25 \%$ and increases the damage state $\boldsymbol{D}$ of a fibre by $25 \%$; this 


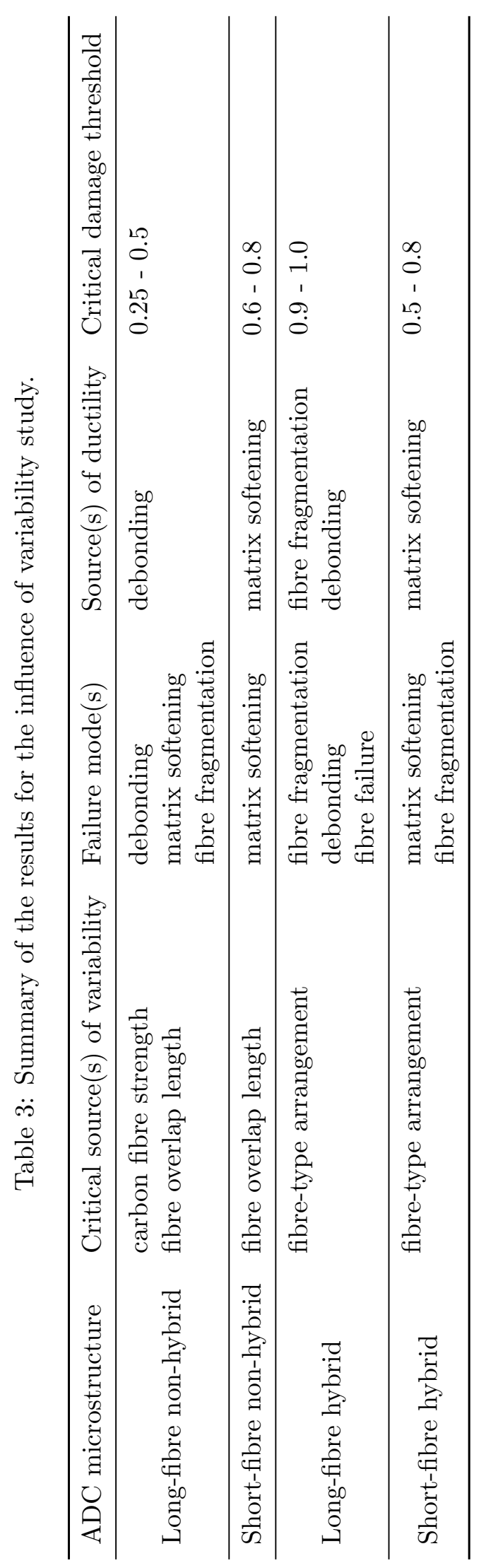


is reflected in Figure 14a by a large spike in the critical damage threshold CDF at 0.25 and 0.50 (corresponding to one or two debonding / fibre fragmentation events respectively).

- Both short-fibre material systems feature widespread matrix softening events (see Figures 11a and 13a, which leads to a progressive increase in the damage state of the fibres as the applied remote strain is increased. This explains why the distribution of the critical damage threshold is quite broad for these material systems (see Figures $14 \mathrm{~b}$ and $14 \mathrm{~d}$ ).

\section{The influence of defects on mechanical performance}

In this section, two different ADC material systems are investigated: defective nonhybrid HSC/EP, and defective hybrid HMC/EG/EP; the material properties and microstructural designs are specified in Table 1 and Table 2 respectively. Different types and amounts of defects were applied to the microstructures and studied using the VTF (as per Section 2.2.2 to investigate the relative influence of these defects.

\subsection{Results}

Figure 15 shows the stress-strain curves for both pristine and defective ADC virtual specimens, for both hybrid and non-hybrid ADC material systems. In both material systems, the $10 \%$ defective virtual specimen contains $10 \%$ fibre fragmentation defects, $10 \%$ interaction defects, and $10 \%$ fibre vacancy defects (where the percentage values are the proportion of total fibres / interactions that were converted into defects, as explained in Section 2.2.2, and likewise for the $25 \%$ defective virtual specimens. While these proportions are arbitrary, a 10\% defective virtual specimen represents a reasonable upper limit of the volume of defects that might be expected in an aerospace- or automotive-grade part. Misalignment was also added to the defective virtual specimens using fibre misalignment data taken from the HiPerDiF method [18] (see Figure 2b). It should be noted that while Figure 15 shows the possibility of a large drop in performance when significant defects are present in ADCs, no significant misalignment, fibre vacancies, or other types of defects were reported in the experimental specimens used for the validation cases in Figure 4. Therefore, the authors estimate the experimental validation cases to have less than $1 \%$ defects, and were thus compared against VTF simulations with no fibre vacancy, fibre 


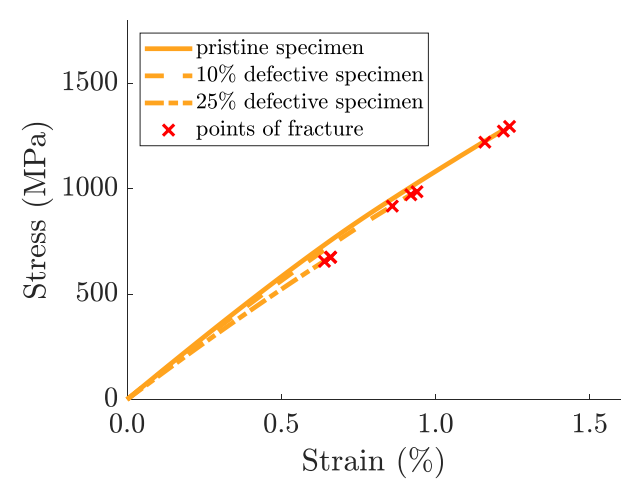

(a) Defective non-hybrid HSC/EP ADC virtual specimens compared to a pristine virtual specimen.

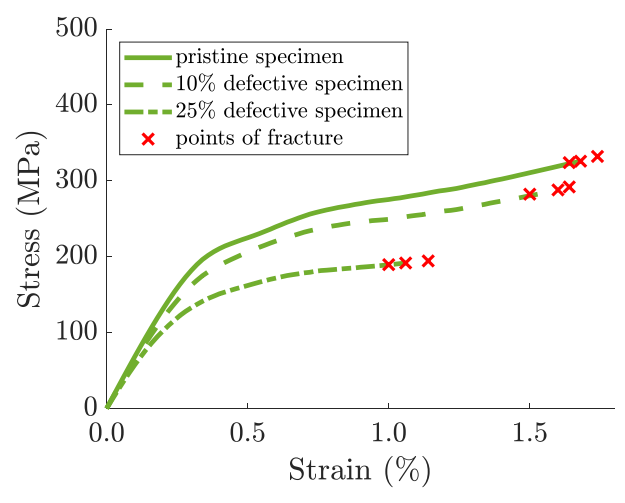

(b) Defective hybrid HMC/EG/EP ADC virtual specimens compared to a pristine virtual specimen.

Figure 15: A greater proportion of defects in the VTF virtual specimens results in a lower initial stiffness and lower ultimate strength when compared to an equivalent pristine virtual specimen. Points of fracture from different simulation runs are shown as a red cross.

interaction, or fibre fragmentation defects, and a small amount of fibre misalignment (as per the HiPerDiF data in Figure 2b.

Next, each of the defect types was applied individually to the non-hybrid HSC/EP and hybrid HMC/EG/EP ADC material systems, in order to quantify the individual contribution of each defect type to the deficit in material performance. The impact of the three different microscale defect types (fibre fragmentation defects, interaction defects, and fibre vacancy defects) on initial stiffness, ultimate strength, and ultimate strain are shown at a range of defect percentages in Figures 16a to 16c, while the overall influence of misalignment on the stress-strain curve of a defective non-hybrid HSC/EP ADC is shown in Figure $16 \mathrm{~d}$.

Finally, the 'heat map' method (as described in Section 3.1.1) was used to determine the relative proportion of each defect type in the critical clusters of 4000 defective virtual specimens, in order to identify which defect type contributes the most to fracture of the hybrid and non-hybrid defective ADC virtual specimens; the results of this study are shown in Figure 17.

\subsection{Discussion}

Several conclusions are drawn from the results in Section 4.1 these are summarised below: 


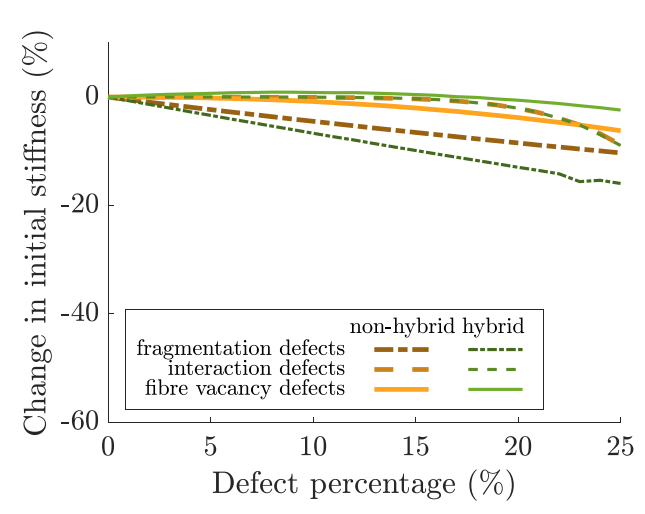

(a) Hybrids and non-hybrids see the same stiffness reduction when interaction defects or fibre vacancy defects are applied, but hybrids see a greater stiffness reduction when fragmentation defects are applied.

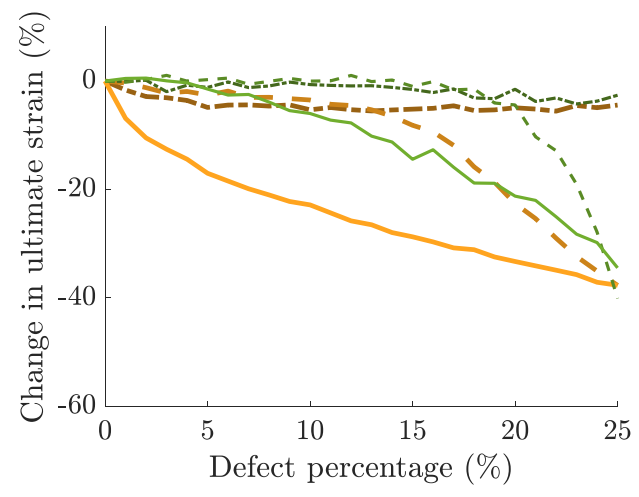

(c) The ultimate strain is affected in a similar way to the ultimate strength of the two material systems, although the ultimate strength of hybrids drops off at a quicker rate than the non-hybrids when the numbers of fibres used for fibre vacancies exceeds $15 \%$.

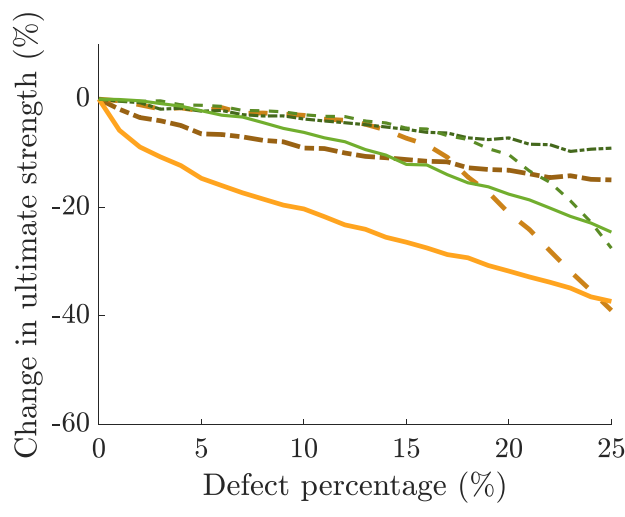

(b) Fibre vacancies have the largest influence on the ultimate strength of ADCs, especially for the non-hybrid material system. Interaction defects have little effect on the ultimate strength until over $15 \%$ of the total number of interfaces are made defective.

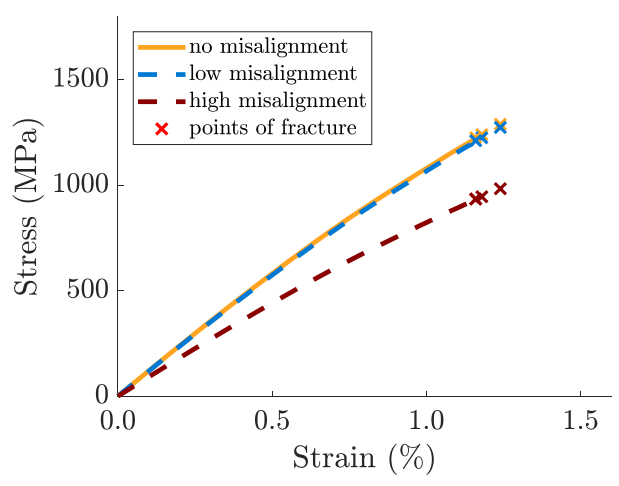

(d) Misalignment has very little influence on the stress-strain curve of the HSC/EP ADC (shown here), and indeed all the material systems considered, provided a process with low fibre misalignment is used. Fibre orientation distributions for the two processes are shown in Figure 2b

Figure 16: The influence of individual types defects on the properties of different material systems (where $\zeta_{\mathrm{rf}}, \zeta_{\mathrm{ri}}$, and $\zeta_{\mathrm{fv}}$ are the fraction of fibre fragmentation, interface, and fibre vacancy defects, respectively). 

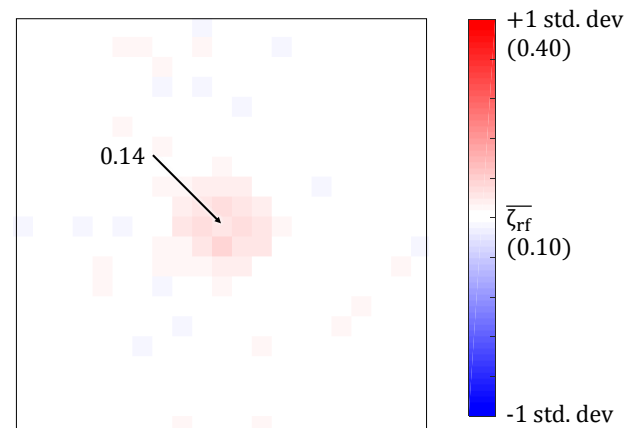

$(-0.20)$

(a) Defective non-hybrid ADC fibre fragmentation defects (where $\zeta_{\mathrm{rf}}$ is the fraction of fibre fragmentation defects).
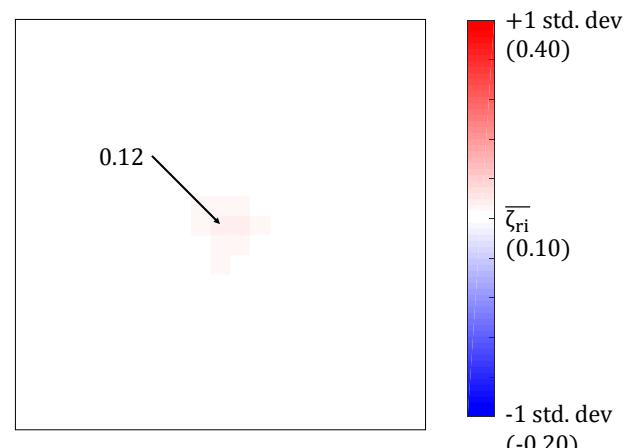

(c) Defective non-hybrid ADC interaction defects (where $\zeta_{\text {ri }}$ is the fraction of interaction defects).
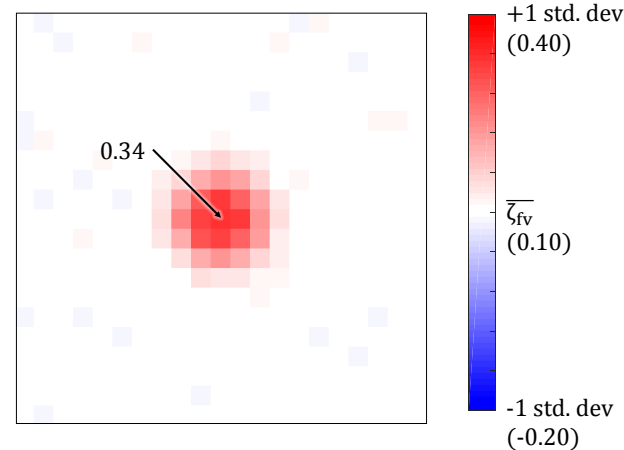

(e) Defective non-hybrid ADC fibre vacancy defects (where $\zeta_{\mathrm{fv}}$ is the fraction of fibre vacancy defects).

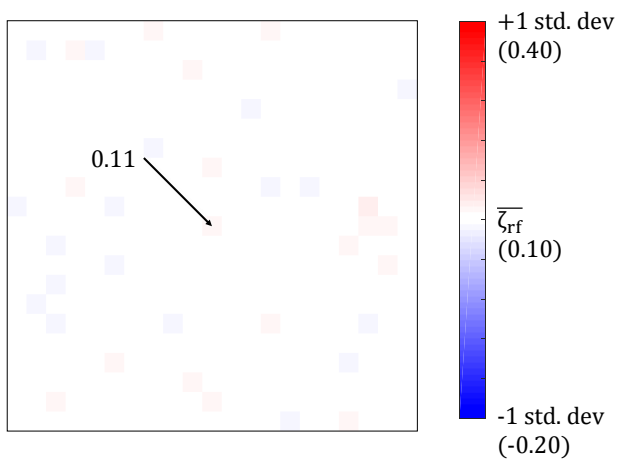

(b) Defective hybrid ADC fibre fragmentation defects (where $\zeta_{\text {rf }}$ is the fraction of fibre fragmentation defects).

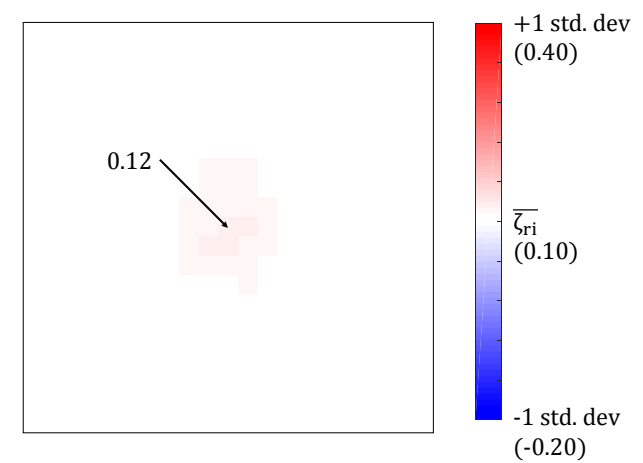

(d) Defective hybrid ADC interaction defects (where $\zeta_{\text {ri }}$ is the fraction of interaction defects).

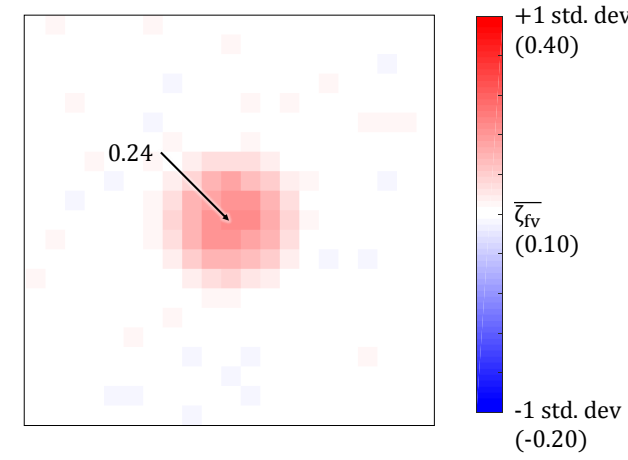

(f) Defective hybrid ADC fibre vacancy defects (where $\zeta_{\mathrm{fv}}$ is the fraction of fibre vacancy defects).

Figure 17: Heat maps showing the concentration of defects around the critical cluster for $10 \%$ defective non-hybrid ADCs (on the left hand side), and $10 \%$ defective hybrid ADCs (on the right hand side). The heat maps are zoomed into a region of 21 by 21 fibres, centred around the critical cluster. 
- Defects significantly reduce the mechanical performance of long-fibre non-hybrid and hybrid ADCs, as demonstrated in Figure 15. The 10\% defective non-hybrid HSC/EP ADC showed a $4 \%$ reduction in initial stiffness, $26 \%$ reduction in ultimate strength, and a $25 \%$ reduction in ultimate strain when compared to a pristine virtual specimen, while the defective hybrid HMC/EG/EP ADC saw a 4\%, 9\%, and 5\% reduction in these properties, respectively.

- Fibre vacancy defects have by far the strongest influence (for a given proportion of defects) on the ultimate strength and ultimate strain of both material systems studied, as shown in Figures 16b to 16c this sensitivity is further substantiated by the high average concentration of fibre vacancy defects in the critical clusters of both material systems (Figure 17). This is because fibre vacancy defects behave like small cracks within the composite (i.e. fibre vacancies have a damage state of $\boldsymbol{D}_{\mathrm{f}}=1$ from the beginning of the virtual test, as per Section 2.2.5), which promote early fracture of the virtual specimen, particularly in regions where multiple fibre vacancy defects coincide.

- Interaction defects only significantly influence the initial stiffness, ultimate strength, and ultimate strain of both materials studied when the percentage of interaction defects is over 15\% (as shown in Figures 16a to 16c); this onset of sensitivity occurs when multiple interaction defects start to act on the same fibre, thus increasing the impact of this defect type.

- Fibre fragmentation defects have a relatively small influence on the ultimate strength and ultimate strain of both material systems studied (as shown in Figures 16b to 16c); this is because while fragmentation defects reduce the overlap lengths of all four interacting fibres, all interactions are still able to transfer load. However, fibre fragmentation defects reduce the aspect ratio of the load-carrying fibres, which reduces the initial stiffness (see Figure 16a); this aspect ratio reduction affects the stiffness of high modulus fibres the most [23], which explains why hybrid HMC/EG material system saw the largest reduction in initial stiffness.

- Defects are less likely to promote premature failure in hybrid ADCs than non-hybrid ADCs, as shown by the lower concentration of defects in the critical clusters of the defective hybrid HMC/EG/EP ADCs (Figure 17), and by the smaller reduction in mechanical properties when compared the pristine material system (shown in 
Figures $16 \mathrm{~b}$ and 16c). This reduced defect sensitivity is because pristine hybrid ADCs undergo large numbers of sub-critical debonding, fibre fragmentation, and

- Additional sources of variability were added to the VTF - including matrix strength variability, inter-fibre distance variability, and fibre modulus variability - now allow the VTF to capture all relevant sources of variability for ADC material systems.

- The ability to model defects, such as residual fibre fragmentations defects, interaction defects, fibre vacancy defects, and fibre misalignment defects. 
- The stress-strain response of two overlapping fibres can now be calculated directly, thus enabling many sources of variability to be considered in the VTF without the use of prohibitively large pre-calculated libraries of overlap stress-strain results.

- A newly-developed fracture mechanics-based failure criterion now accounts not only for the damage due to fibre failure, but all forms of relevant damage present in ADCs (including contributions from matrix softening / debonding, fibre fibre fragmentation, and defects); this fracture criterion enabled successful validation of the VTF against a large variety of experimental test cases.

- Various damage events, such as matrix softening, matrix debonding, fibre fragmentation, and fibre failure, can now be identified and tracked during virtual tests; this gives further insight into how damage develops in each material system until final failure.

After performing the analysis of several ADC material system with the developed virtual testing framework, the following conclusions were drawn:

- Property 'heat maps' can be used to identify the critical source of variability in each material system; it is proven that the critical source of variability (i.e. the source of variability that contributed most to final failure) changes with each material system. Long-fibre non-hybrid aligned discontinuous composites are most sensitive to fibre strength variability and the fibre overlap length, short-fibre non-hybrid aligned discontinuous composites are most susceptible to the fibre overlap length, and failure of hybrid aligned discontinuous composite material systems is dominated by the fibre-type arrangement (regardless of fibre length).

- Fibre vacancy (or voidage) defects are a more critical defect type than fibre fragmentation defects, interaction defects, or fibre misalignment defects; in other words, fibre vacancy defects most readily promote premature final failure in short- and long-fibre hybrid and non-hybrid ADCs.

- In general, hybrid ADCs are less sensitive to defects than non-hybrid ADCs; this reduced sensitivity is because pristine hybrid ADCs often already experience a large number of sub-critical damage events before failing, which makes the effects of the defects considered in this study less pronounced. 
- Misalignment has a significant influence on the stress-strain response of ADCs when the average fibre misalignment is high; however, the influence of misalignment can be reduced through the use of high quality fibre-alignment techniques such as the HiPerDiF method [18]. The differences in performance between these two alignment cases will be more significant if the influence of fibre misalignment on the fibre volume fraction is also considered.

The developed virtual testing framework is proven to be a robust and complete tool for the design of aligned discontinuous composites, and the authors welcome other researchers to use this virtual testing framework to design new materials or explore other avenues of research. A live GitHub repository of the working code can be found here: https://github. com/meComposites/Aligned-Discontinuous-Composites/releases/tag/The_influen_e_

of_variability_and_defects.

\section{Acknowledgements}

This work was funded under the UK Engineering and Physical Sciences Research Council (EPSRC) programme grant EP/I02946X/1 on High Performance Ductile Composite Technology in collaboration with the University of Bristol. S. Pimenta acknowledges the support from the Royal Academy of Engineering for her Research Fellowship on Multiscale discontinuous composites for large scale and sustainable structural applications (20152019). The authors would also like to thank Marco L. Longana (University of Bristol) and $\mathrm{HaNa} \mathrm{Yu}$ (University of Bath) for the experimental results used in this paper.

All underlying data to support the conclusions are provided within this paper.

\section{References}

[1] Netravali AN, Chabba S. Composites get greener. Materials Today. 2003 apr;6(4):2229.

[2] Gusev AA, Hine PJ, Ward IM. Fiber packing and elastic properties of a transversely random unidirectional glass/epoxy composite. Compos Sci Technol. 2000 mar;60(4):535-541.

[3] Sanei SHR, Fertig RS. Uncorrelated volume element for stochastic modeling of microstructures based on local fiber volume fraction variation. Compos Sci Technol. 2015 sep;117:191-198. 
[4] Wisnom MR. Relationship between strength variability and size effect in unidirectional carbon fibre/epoxy. Composites. 1991 jan;22(1):47-52.

[5] Lekou DJ, Philippidis TP. Mechanical property variability in FRP laminates and its effect on failure prediction. Composites Part B. 2008;39(7-8):1247-1256.

[6] Netravali AN, Henstenburg RB, Phoenix SL, Schwartz P. Interfacial shear strength studies using the single filament composite test. I: Experiments on graphite fibers in epoxy. Polym Compos. 1989 aug;10(4):226-241.

[7] Chen C, Zhang C, Zhao Z, Wang Y, Wong SC, Li Y. Effect of fiber reinforcement and fabrication process on the dynamic compressive behavior of PEEK composites. Int J Mech Sci. 2019;155:170-177.

[8] Potter KD, Khan B, Wisnom MR, Bell T, Stevens J. Variability, fibre waviness and misalignment in the determination of the properties of composite materials and structures. Composites Part A. 2008;39(9):1343-1354.

[9] Bowles KJ, Frimpong S. Void effects on the interlaminar shear strength of unidirectional graphite-fiber-reinforced composites. J Compos Mater. 1992 oct;26(10):14871509.

[10] De Almeida SFM, Neto ZdSN. Effect of void content on the strength of composite laminates. Compos Struct. 1994 jan;28(2):139-148.

[11] Ostoja-Starzewski M. Material spatial randomness: From statistical to representative volume element. Probabilistic Engineering Mechanics. 2006;21(2):112-132.

[12] Terada K, Miura T, Kikuchi N. Digital image-based modeling applied to the homogenization analysis of composite materials. Comput Mech. 1997;20:331-346.

[13] Shan Z, Gokhale AM. Digital image analysis and microstructure modeling tools for microstructure sensitive design of materials. Int J Plast. 2004 jul;20(7):1347-1370.

[14] Van Vinckenroy G, de Wilde WP. The use of Monte Carlo techniques in statistical finite element methods for the determination of the structural behaviour of composite materials structural components. Compos Struct. 1995 jan;32(1-4):247-253.

[15] Liu X, Mahadevan S, Pradhan B, Kumar S. Ultimate strength failure probability estimation of composite structures. J Reinf Plast Compos. 2000 mar;19(5):403-426. 
[16] Sriramula S, Chryssanthopoulos MK. Quantification of uncertainty modelling in stochastic analysis of FRP composites. Composites Part A. 2009 nov;40(11):16731684 .

[17] Said BE, Hallett SR. Multiscale surrogate modelling of the elastic response of thick composite structures with embedded defects and features. Compos Struct. 2018 may;200:781-798.

[18] Yu H, Potter KD, Wisnom MR. A novel manufacturing method for aligned discontinuous fibre composites (High Performance-Discontinuous Fibre method). Composites Part A. 2014;65:175-185.

[19] Henry J, Pimenta S. Semi-analytical simulation of aligned discontinuous composites. Compos Sci Technol. 2017;144:230-244.

[20] Pimenta S, Robinson P. An analytical shear-lag model for composites with brickand-mortar architecture considering non-linear matrix response and failure. Compos Sci Technol. 2014 nov;104:111-124.

[21] Czél G, Pimenta S, Wisnom MR, Robinson P. Demonstration of pseudo-ductility in unidirectional discontinuous carbon fibre/epoxy prepreg composites. Compos Sci Technol. 2015;106:110-119.

[22] Yu H, Longana ML, Jalalvand M, Wisnom MR, Potter KD. Pseudo-ductility in intermingled carbon/glass hybrid composites with highly aligned discontinuous fibres. Composites Part A. 2015;73:35-44.

[23] Henry J, Pimenta S. Modelling hybrid effects on the stiffness of aligned discontinuous composites with hybrid fibre-types. Compos Sci Technol. 2017;152(1):275-289.

[24] Henry J, Pimenta S. Virtual testing framework for hybrid aligned discontinuous composites. Compos Sci Technol. 2018 may;159:259-272.

[25] Finley JM, Yu H, Longana ML, Pimenta S, Shaffer MSP, Potter KD. Exploring the pseudo-ductility of aligned hybrid discontinuous composites using controlled fibretype arrangements. Composites Part A. 2017 apr;107:592-606.

[26] Swolfs Y, Gorbatikh L, Verpoest I. Fibre hybridisation in polymer composites: A review. Composites Part A. 2014;67:181-200. 
[27] Jalalvand M, Czél G, Wisnom MR. Damage analysis of pseudo-ductile thin-ply UD hybrid composites A new analytical method. Composites Part A. 2015;69:83-93.

[28] Jalalvand M, Czél G, Wisnom MR. Parametric study of failure mechanisms and optimal configurations of pseudo-ductile thin-ply UD hybrid composites. Composites Part A. 2015;74:123-131.

[29] Pimenta S, Pinho ST. The influence of micromechanical properties and reinforcement architecture on the mechanical response of recycled composites. Composites Part A. 2014 jan;56:213-225.

[30] Johnson DJ. Structure-property relationships in carbon fibres. J Phys D. 1987;20(3):286-291.

[31] Billingsley P. The central limit theorem. In: Probability and Measure. 3rd ed. New York: J. Wiley; 1995. p. 357-370.

[32] Swolfs Y, Gorbatikh L, Romanov V, Orlova S, Lomov SV, Verpoest I. Stress concentrations in an impregnated fibre bundle with random fibre packing. Compos Sci Technol. 2013 jan;74:113-120.

[33] Van Den Heuvel PWJ, Peijs T, Young RJ. Failure phenomena in two-dimensional multi-fibre microcomposites: 2. A Raman spectroscopic study of the influence of interfibre spacing on stress concentrations. Compos Sci Technol. 1997 jan;57(8):899-911.

[34] St-Pierre L, Martorell NJ, Pinho ST. Stress redistribution around clusters of broken fibres in a composite. Compos Struct. 2017 may;168:226-233.

[35] Collins R. A geometrical sum rule for two-dimensional fluid correlation functions. J Phys C. 1968;1(6):1461-1471.

[36] Fu SY, Hu X, Yue CY. The flexural modulus of misaligned short-fiber-reinforced polymers. Compos Sci Technol. 1999;59(10):1533-1542.

[37] Butcher JC. The Euler method. In: Numerical Methods for Ordinary Differential Equations. 2nd ed. New Jersey: J. Wiley; 1999. p. 51-91.

[38] Sanadi A. Interfacial effects in carbon-epoxies. J Mat Sci. 1985;20(2):421 - 430. 
[39] Scott AE, Mavrogordato M, Wright P, Sinclair I, Spearing SM. In situ fibre fracture measurement in carbon-epoxy laminates using high resolution computed tomography. Compos Sci Technol. 2011 aug;71(12):1471-1477.

[40] MathWorks. bwconncomp - MATLAB R2018a documentation; 2018. [Online; accessed 14-May-2018]. Available from: https://uk.mathworks.com/help/images/ ref/bwconncomp.html.

[41] De Geus TWJ, Peerlings RHJ, Geers MGD. Microstructural topology effects on the onset of ductile failure in multi-phase materials - a systematic computational approach. Int J Solids Struct. 2015 aug;67-68:326-339.

[42] Muche L. The Poisson Voronoi tessellation III. Miles' formula. Mathematische Nachrichten. 1998;191(1):247-267.

[43] Swolfs Y, Gorbatikh L, Verpoest I. Stress concentrations in hybrid unidirectional fibre-reinforced composites with random fibre packings. Compos Sci Technol. 2013 aug;85:10-16.

\section{Appendices}

\section{Appendix A Derivation of the inter-fibre distance load-sharing coefficient}

The Poission-Voronoi Tesselation Process (PVTP) is commonly used to sample the distance between neighbouring points that are randomly located in space [35, 42]; in all cases the distance between neighbouring points must be positive. This appendix explains how the PVTP is adapted to sample the inter-fibre distance in a composite virtual specimen, within the scope of the VTF. This distribution of inter-fibre distances must be compatible with the fibre volume fraction of the composite material, which can be calculated as

$$
v_{\mathrm{f}}=\frac{\mathbb{E}\left(\phi_{\mathrm{f}}^{2}\right)}{\mathbb{E}\left(\phi_{\mathrm{u}}^{2}\right)}=\frac{\mathbb{E}\left(\phi_{\mathrm{f}}^{2}\right)}{\mathbb{E}\left(\phi_{\mathrm{f}}^{2}\right)+2 \cdot \mathbb{E}\left(\phi_{\mathrm{f}}\right) \cdot \mathbb{E}\left(t_{\mathrm{i}}\right)+\mathbb{E}\left(t_{\mathrm{i}}^{2}\right)},
$$

where $\phi_{\mathrm{f}}$ is the fibre diameter, $\phi_{\mathrm{u}}=\phi_{\mathrm{f}}+t_{\mathrm{i}}$ is the external diameter of a cylindrical unit-cell of the composite, and $\mathbb{E}\left(t_{\mathrm{i}}\right)$ is the expected value of the inter-fibre distance for the virtual specimen. 
For the application of variable inter-fibre distances, the PVTP is used to sample the distance between the outer diameters of neighbouring fibres ( $t_{\mathrm{i}_{N}}$ in Figure $\left.1 \mathrm{~b}\right)$. By sampling the distance between the outer diameter of the fibres (and not the distance between the fibre centres), the PVTP ensures that no neighbouring fibres intersect one-another. Distances between neighbouring fibres are calculated by sampling the cumulative distribution function $(\mathrm{CDF})$ of inter-fibre distances, which is generated according to the following function (derived from work by Collins [35] and Muche [42]):

$$
\operatorname{CDF}\left(t_{\mathrm{i}_{N}}\right)=1-\left(\frac{1-\pi \cdot p \cdot t_{\mathrm{i}_{N}}}{6}\right) \cdot \operatorname{erfc}\left(\frac{\sqrt{\pi \cdot p} \cdot t_{\mathrm{i}_{N}}}{2}\right)-\left(\sqrt{p} \cdot t_{\mathrm{i}_{N}}\right) \cdot \exp \left(\frac{-\pi \cdot p \cdot{t_{\mathrm{i}_{N}}}^{2}}{4}\right),
$$

where erfc is the residual error function, and $p$ is the equivalent areal density of the PVTP, which is calculated such that the volume fraction and the expected inter-fibre distance are consistent with Equation 3 (in which the distribution of $t_{\mathrm{i}}$ is the only unknown):

$$
p=\frac{1024 \cdot \mathbb{E}\left(t_{\mathrm{i}}\right)^{2}}{81 \cdot \pi^{2}}
$$

St-Pierre et al. 34 demonstrated that the Stress Concentration Factor (SCF) experienced by the $N^{\text {th }}$ fibre neighbouring a central fibre-break or fibre-end is proportional to a power law applied to the fibre-centre distance:

$$
\mathrm{SCF}_{N}=1+\lambda \cdot\left(\frac{\phi_{\mathrm{f}_{\mathrm{a}}}}{t_{\mathrm{a}_{N}}}\right)^{2}
$$

where $\lambda$ is the total load coefficient (which can be determined by equilibrium), $\phi_{\mathrm{f}_{\mathrm{a}}}$ is the central fibre diameter, and $t_{\mathrm{a}_{N}}$ is the fibre-centre distance (see Figure 1b), which can be calculated as the distance between the centre of the $N^{\text {th }}$ neighbouring fibre to the outer diameter of the central fibre:

$$
t_{\mathrm{a}_{N}}=t_{\mathrm{i}_{N}}+\frac{\phi_{\mathrm{f}_{N}}}{2} .
$$

The overload experienced by a fibre neighbouring a break in a non-hybrid composite is

$$
\Delta F_{N}=\left(\mathrm{SCF}_{N}-1\right) \cdot \varepsilon^{\infty} \cdot E_{\mathrm{f}_{\mathrm{a}}} \cdot A_{\mathrm{f}_{\mathrm{a}}},
$$

where $E_{\mathrm{f}_{\mathrm{a}}}$ and $A_{\mathrm{f}_{\mathrm{a}}}$ are the Young's modulus and cross-section area of the fibre respectively. Therefore, Equations 6 and 8 suggest that the overload experienced by the neighbouring fibre is inversely proportional to $t_{\mathrm{a}_{N}}^{2}$. 
In the shear-lag model for discontinuous composites [19], a fibre neighbouring a fibrebreak/discontinuity will experience an overload of

$$
\Delta F_{N}=\varepsilon^{\infty} \cdot E_{\mathrm{f}_{\mathrm{a}}} \cdot \frac{\pi \cdot \phi_{\mathrm{f}_{\mathrm{a}}}}{4} \cdot T_{\mathrm{a}_{N}},
$$

where $T_{\mathrm{a}_{N}}$ is the characteristic thickness of the broken/discontinuous fibre in that fibrefibre interaction. Consequently, by combining Equations 8 and 9 above, one can mimick the power law in Equation 6 in the shear-lag model for discontinuous composites by setting $T_{\mathrm{a}_{N}} \propto 1 / t_{\mathrm{a}_{N}}^{2}$. Moreover, since $\sum_{N=1}^{N *} T_{\mathrm{a}_{N}}=\phi_{\mathrm{f}_{\mathrm{a}}}$ in the shear-lag model [19], then the characteristic thickness can be defined as

$$
T_{\mathrm{a}_{N}}=\alpha / t_{\mathrm{a}_{N}}^{2},
$$

where the load-sharing coefficient $\alpha$ is defined as

$$
\alpha=\frac{\phi_{\mathrm{f}_{\mathrm{a}}}}{\sum_{N=1}^{N *} \frac{1}{t_{\mathrm{a}_{N}}^{2}}} .
$$

This methodology was based on studies of the load-sharing near fibre-breaks in nonhybrid composites [34] (see Equation 6). In order to verify its consistency in the case of hybrid reinforcement, let us consider stress concentration factors for fibres surrounding a fibre break, calculated by Swolfs et al. 43 via 3D finite element simulations. Three different hybrid microstructures were analysed, with different combinations of fibre areas and fibre Young's moduli, as summarised in Table4. For the two cases where the structural stiffnesses (i.e. the product of the fibre Young's modulus $E_{\mathrm{f}}$ and fibre cross-sectional area $A_{\mathrm{f}}$ ) of the neighbouring fibres are approximately equal to that of the central fibre, a very similar stress concentration factor, and overload $\Delta F_{N}$, was observed (for a given inter-fibre distance); for the case where the structural stiffnesses of the neighbouring fibres differed by a factor of three, an approximate three times increase in stress concentration factor was observed, while the overload remained constant (because the change in load is the same across all fibres [43]). This suggests that the same overload $\Delta F_{N}$ is experienced by all $N^{*}$ neighbouring fibres, regardless of the differences in fibre diameter or Young's modulus, provided the neighbouring fibres are at the same inter-fibre distance. Therefore, the derivation of the load-sharing method derived in Equations 6 to 11 is applicable to the case of both non-hybrid and hybrid reinforcements. 
Table 4: The relative difference in stress concentration factors and overload values (at a given inter-fibre distance) for three different fibre combinations, according to Swolfs et al. [43].

\begin{tabular}{|c|c|c|c|c|c|c|}
\hline $\begin{array}{l}\text { Central fibre } \\
\text { (subscript a) }\end{array}$ & $\begin{array}{l}\text { Neighbouring fibre } \\
(\text { subscript } N)\end{array}$ & $\frac{A_{\mathrm{f}_{\mathrm{c}}}}{A_{\mathrm{fg}_{\mathrm{g}}}}$ & $\frac{E_{\mathrm{f}_{\mathrm{c}}}}{E_{\mathrm{f}_{\mathrm{g}}}}$ & $\frac{A_{\mathrm{f}_{\mathrm{c}}} \cdot E_{\mathrm{f}_{\mathrm{c}}}}{A_{\mathrm{fg}_{\mathrm{g}}} \cdot E_{\mathrm{fg}_{\mathrm{g}}}}$ & $\mathrm{SCF}_{N}-1$ & $\begin{array}{l}\text { Overload } \\
\left(\Delta F_{N}\right)\end{array}$ \\
\hline Carbon & Carbon & 1.00 & 1.00 & 1.00 & baseline value & baseline value \\
\hline Carbon & Glass (equal diameter) & 1.00 & 3.33 & 3.33 & approx. $3 \times$ baseline & approx. $1 \times$ baseline \\
\hline Carbon & Glass (larger diameter) & 0.34 & 3.33 & 1.13 & approx. $1 \times$ baseline & approx. $1 \times$ baseline \\
\hline
\end{tabular}

\section{Appendix B Scaling the volume fraction and carbon ratio for fibre vacancy defects}

Fibre vacancy defects are implemented in the VTF by setting the stress-strain curves of a specified number of fibres (and their neighbouring fibre-fibre interactions) to zero at the beginning of the virtual test (as mentioned in Section 2.2.2), thus making the fibre behave like a fibre vacancy (or void). Since the number of active fibres should be maintained in the cross-section of every RVE according to the inputs specified in Table 2, the total number of fibres included in the cross-section at the beginning of the virtual test should be the sum of the number of active fibres, plus the number of fibre vacancy defects. The total number of carbon and glass fibres (including fibre vacancy defects) are therefore calculated as

$$
\begin{gathered}
n_{\mathrm{f}_{\mathrm{c}}}^{\S}=\left(1+\zeta_{\mathrm{fv}_{\mathrm{c}}}\right) n_{\mathrm{f}_{\mathrm{c}}}, \text { and } \\
n_{\mathrm{fg}_{\mathrm{g}}}^{\S}=\left(1+\zeta_{\mathrm{fvg}_{\mathrm{g}}}\right) n_{\mathrm{fg}_{\mathrm{g}}},
\end{gathered}
$$

where $n_{\mathrm{f}_{\mathrm{c}}}^{\S}$ and $n_{\mathrm{f}_{\mathrm{g}}}^{\S}$ are the total number of carbon and glass fibres (including those used to simulate fibre vacancies), $\zeta_{\mathrm{fv}_{\mathrm{c}}}$ and $\zeta_{\mathrm{fvg}_{\mathrm{g}}}$ are the numeric proportion of carbon and glass fibres that are used to simulate fibre vacancy defects, and $n_{\mathrm{f}_{\mathrm{c}}}$ and $n_{\mathrm{fg}}$ are the number of active carbon and glass fibres (i.e. the fibres that are used to transfer load in the virtual specimen), respectively.

Care must also be taken to ensure that the aspect ratio of the virtual specimen crosssection is preserved. The aspect ratio of the cross-section is defined as

$$
\mathrm{AR}=\frac{n_{\mathrm{f}_{\mathrm{j}}}}{n_{\mathrm{f}_{\mathrm{i}}}}=\frac{n_{\mathrm{f}_{\mathrm{j}}}^{\S}}{n_{\mathrm{f}_{\mathrm{i}}}^{\S}},
$$

where $n_{\mathrm{f}_{\mathrm{i}}}$ is the original number of fibres along the height of the virtual specimen cross- 
section, $n_{\mathrm{f}_{\mathrm{j}}}$ is the original number of fibres across the width, and $n_{\mathrm{f}_{\mathrm{i}}}^{\S}$ and $n_{\mathrm{f}_{\mathrm{j}}}^{\S}$ are the number of fibres across the cross-section width and height when fibre vacancy defects are included. Scaling the total number of fibres (including fibre vacancy defects) while maintaining the aspect ratio of the pristine specimen yields

$$
\begin{gathered}
n_{\mathrm{f}_{\mathrm{i}}}^{\S}=\operatorname{round}\left(\sqrt{\left(n_{\mathrm{f}_{\mathrm{c}}}^{\S}+n_{\mathrm{f}_{\mathrm{g}}}^{\S}\right) \cdot \frac{n_{\mathrm{f}_{\mathrm{i}}}}{n_{\mathrm{f}_{\mathrm{j}}}}}\right), \text { and } \\
n_{\mathrm{f}_{\mathrm{j}}}^{\S}=\operatorname{round}\left(\sqrt{\left(n_{\mathrm{f}_{\mathrm{c}}}^{\S}+n_{\mathrm{f}_{\mathrm{g}}}^{\S}\right) \cdot \frac{n_{\mathrm{f}_{\mathrm{j}}}}{n_{\mathrm{f}_{\mathrm{i}}}}}\right) .
\end{gathered}
$$

The input fibre volume fraction $v_{\mathrm{f}}$ must also be corrected to account for both the volume of the active fibres and the volume of the fibre vacancies. The corrected volume fraction $v_{\mathrm{f}}^{\S}$ (i.e. the fibre volume fraction including the additional fibre vacancies) is calculated such that the fibre volume fraction of active fibres within the defective virtual specimen matches that of a pristine specimen:

$$
v_{\mathrm{f}}^{\S}=v_{\mathrm{f}}\left(1+\frac{\zeta_{\mathrm{fv}_{\mathrm{c}}} \cdot n_{\mathrm{f}_{\mathrm{c}}} \cdot \phi_{\mathrm{f}_{\mathrm{c}}}^{2}+\zeta_{\mathrm{fv}_{\mathrm{g}}} \cdot n_{\mathrm{fg}_{\mathrm{g}}} \cdot \phi_{\mathrm{f}_{\mathrm{g}}}^{2}}{n_{\mathrm{f}_{\mathrm{c}}} \cdot \phi_{\mathrm{f}_{\mathrm{c}}}^{2}+n_{\mathrm{fg}_{\mathrm{g}}} \cdot \phi_{\mathrm{f}_{\mathrm{g}}}^{2}}\right) .
$$

Finally, the corrected carbon ratio (i.e. the volumetric proportion of carbon fibres, corrected to include all fibres used to simulate fibre vacancy defects) is calculated such that the volume ratio of active carbon fibres to active glass fibres is consistent with that of the carbon ratio $v_{\mathrm{c}}$ of a pristine specimen:

$$
v_{\mathrm{c}}^{\S}=\frac{\left(1+\zeta_{\mathrm{fv}_{\mathrm{c}}}\right) \cdot n_{\mathrm{f}_{\mathrm{c}}} \cdot \phi_{\mathrm{f}_{\mathrm{c}}}^{2}}{\left(1+\zeta_{\mathrm{fv}_{\mathrm{c}}}\right) \cdot n_{\mathrm{f}_{\mathrm{c}}} \cdot \phi_{\mathrm{f}_{\mathrm{c}}}^{2}+\left(1+\zeta_{\mathrm{fvg}_{\mathrm{g}}}\right) \cdot n_{\mathrm{fg}_{\mathrm{g}}} \cdot \phi_{\mathrm{fg}_{\mathrm{g}}}^{2}} .
$$

